\title{
MINIMAL REGULARITY SOLUTIONS OF SEMILINEAR GENERALIZED TRICOMI EQUATIONS
}

\author{
ZHUOPING RUAN, INGO WITT, AND HUICHENG YIN
}

\begin{abstract}
We prove the local existence and uniqueness of minimal regularity solutions $u$ of the semilinear generalized Tricomi equation $\partial_{t}^{2} u-t^{m} \Delta u=F(u)$ with initial data $\left(u(0, \cdot), \partial_{t} u(0, \cdot)\right) \in$ $\dot{H^{\gamma}}\left(\mathbb{R}^{n}\right) \times \dot{H}^{\gamma-\frac{2}{m+2}}\left(\mathbb{R}^{n}\right)$ under the assumption that $|F(u)| \lesssim|u|^{\kappa}$ and $\left|F^{\prime}(u)\right| \lesssim|u|^{\kappa-1}$ for some $\kappa>1$. Our results improve previous results of M. Beals [2] and of ourselves [15-17]. We establish Strichartz-type estimates for the linear generalized Tricomi operator $\partial_{t}^{2}-t^{m} \Delta$ from which the semilinear results are derived.
\end{abstract}

\section{INTRODUCTION}

In this paper, we are concerned with the local well-posedness problem for minimal regularity solutions $u$ of the semilinear generalized Tricomi equation

$$
\left\{\begin{array}{l}
\partial_{t}^{2} u-t^{m} \Delta u=F(u) \quad \text { in }(0, T) \times \mathbb{R}^{n}, \\
u(0, \cdot)=\varphi \in \dot{H}^{\gamma}\left(\mathbb{R}^{n}\right), \\
\partial_{t} u(0, \cdot)=\psi \in \dot{H}^{\gamma-\frac{2}{m+2}}\left(\mathbb{R}^{n}\right),
\end{array}\right.
$$

where $n \geq 2, m \in \mathbb{N}, \gamma \in \mathbb{R}, \Delta=\sum_{i=1}^{n} \partial_{i}^{2}$, and $T>0$. The nonlinearity $F \in C^{1}(\mathbb{R})$ obeys the estimates

$$
|F(u)| \lesssim|u|^{\kappa}, \quad\left|F^{\prime}(u)\right| \lesssim|u|^{\kappa-1}
$$

for some $\kappa>1$. For $n \geq 3$ and $\kappa>\kappa_{3}$ (see below) we further assume that $\kappa \in \mathbb{N}$ and $F(u)= \pm u^{\kappa}$.

Our main objective of this paper is to find the minimal number $\gamma$ for which Eq. (1.1) under assumption (1.2) possesses a unique local solution $u \in C\left([0, T], \dot{H}^{\gamma}\left(\mathbb{R}^{n}\right)\right) \cap L^{s}\left((0, T) ; L^{q}\left(\mathbb{R}^{n}\right)\right)$ for certain $s, q$ with $\min \{s, q\} \geq \kappa$. Then $F(u) \in L^{s / \kappa}\left((0, T) ; L^{q / \kappa}\left(\mathbb{R}^{n}\right)\right) \subseteq L_{\mathrm{loc}}^{1}\left((0, T) \times \mathbb{R}^{n}\right)$, and Eq. (1.1) holds in distributions.

We first introduce notation used throughout this paper. Set

$$
\begin{array}{cr}
\mu_{*}=\frac{(m+2) n+2}{2}, \quad \kappa_{*}=\frac{\mu_{*}+2}{\mu_{*}-2}=\frac{(m+2) n+6}{(m+2) n-2}, \\
\kappa_{0}=1+\frac{6 \mu_{*}+m}{\mu_{*}(m+2) n} & \text { if } n \geq 3 \text { or } n=2, m \geq 3,
\end{array}
$$

2010 Mathematics Subject Classification. Primary: 35L70; Secondary: 35L65, 35L67, 76N15.

Key words and phrases. Generalized Tricomi equation, minimal regularity, Fourier integral operators, Stricharz estimates.

The first and third authors were supported by the NSFC (No. 11401299, No. 11571177) and by the Priority Academic Program Development of Jiangsu Higher Education Institutions. 


$$
\begin{aligned}
& \kappa_{1}= \begin{cases}2 & \text { if } n=2, m=1, \\
\frac{\left(\mu_{*}+2\right)(m+2)(n-1)+8}{\left(\mu_{*}-2\right)(m+2)(n-1)+8} & \text { if } n \geq 3 \text { or } n=2, m \geq 2,\end{cases} \\
& \kappa_{2}=\frac{\mu_{*}\left(\mu_{*}+2\right)(n-1)-2(n+1)}{\mu_{*}\left(\mu_{*}-2\right)(n-1)-2(n+1)},
\end{aligned}
$$

and

$$
\kappa_{3}=\frac{\mu_{*}-m}{\mu_{*}-m-4} \quad \text { if } n \geq 3 .
$$

Note that $\mu_{*}$ is the homogeneous dimension of the degenerate differential operator $\partial_{t}^{2}-t^{m} \Delta$ and $\kappa_{*}$ is the power $\kappa$ for which the equation $\partial_{t}^{2} u-t^{m} \Delta u= \pm|u|^{\kappa-1} u$ is conformally invariant. Note further that $1<\kappa_{0}<\kappa_{1}<\kappa_{*}<\kappa_{2}<\kappa_{3}$ whenever it applies.

Now we state the main results of this paper.

Theorem 1.1. Let $n \geq 2$ and $F$ be as above. Suppose further $\kappa>\kappa_{1}$ and $(\varphi, \psi) \in \dot{H}^{\gamma}\left(\mathbb{R}^{n}\right) \times$ $\dot{H}^{\gamma-\frac{2}{m+2}}\left(\mathbb{R}^{n}\right)$, where

$$
\gamma=\gamma(\kappa, m, n)= \begin{cases}\frac{n+1}{4}-\frac{n+1}{\mu_{*}(\kappa-1)}-\frac{m}{2 \mu_{*}(m+2)} & \text { if } \kappa_{1}<\kappa \leq \kappa_{*}, \\ \frac{n}{2}-\frac{4}{(m+2)(\kappa-1)} & \text { if } \kappa \geq \kappa_{*} .\end{cases}
$$

Then problem (1.1) possesses a unique solution

$$
u \in C\left([0, T] ; \dot{H}^{\gamma}\left(\mathbb{R}^{n}\right)\right) \cap L^{s}\left((0, T) ; L^{q}\left(\mathbb{R}^{n}\right)\right)
$$

for some $T>0$, where

$$
\|u\|_{C\left([0, T] ; \dot{H}^{\gamma}\left(\mathbb{R}^{n}\right)\right)}+\|u\|_{L^{s}\left((0, T) ; L^{q}\left(\mathbb{R}^{n}\right)\right)} \lesssim\|\varphi\|_{\dot{H}^{\gamma}\left(\mathbb{R}^{n}\right)}+\|\psi\|_{\dot{H}^{\gamma-\frac{2}{m+2}\left(\mathbb{R}^{n}\right)}}
$$

and $q=\mu_{*}(\kappa-1) / 2$,

$$
\frac{1}{s}= \begin{cases}\frac{(m+2)(n-1)}{4}\left(\frac{1}{2}-\frac{1}{q}\right)+\frac{m}{4 \mu_{*}} & \text { if } \kappa_{1}<\kappa \leq \kappa_{*}, \\ 1 / q & \text { if } \kappa \geq \kappa_{*} .\end{cases}
$$

Remark 1.2. As a byproduct of the proof of Theorem 1.1, we see that problem (1.1) admits a unique global solution $u \in C\left([0, \infty) ; \dot{H}^{\gamma}\left(\mathbb{R}^{n}\right)\right) \cap L^{\infty}\left((0, \infty) ; \dot{H}^{\gamma}\left(\mathbb{R}^{n}\right)\right) \cap L^{\frac{\mu_{*}(\kappa-1)}{2}}\left(\mathbb{R}_{+} \times \mathbb{R}^{n}\right)$ in case $n \geq 2$, $\kappa \geq \kappa_{*}$ if $(\varphi, \psi)=\varepsilon\left(u_{0}, u_{1}\right),\left(u_{0}, u_{1}\right) \in \dot{H}^{\gamma}\left(\mathbb{R}^{n}\right) \times \dot{H}^{\gamma-\frac{2}{m+2}}\left(\mathbb{R}^{n}\right)$, and $\varepsilon>0$ is small (cf. 5.1.2 and 5.1.3 in the proof of Theorem 1.1 below). With a different argument, the global result $u \in$ $L^{\frac{\mu_{*}(\kappa-1)}{2}}\left(\mathbb{R}_{+} \times \mathbb{R}^{n}\right)$ for problem (1.1) was obtained in [7].

Remark 1.3. For $\gamma<\frac{n}{2}-\frac{4}{(m+2)(\kappa-1)}$, one obtains ill-posedness for problem (1.1) by scaling. More specifically, if $u=u(t, x)$ solves the Cauchy problem (1.1), where $F(u)= \pm|u|^{\kappa-1} u$, then

$$
u_{\varepsilon}(t, x)=\varepsilon^{-\frac{2}{\kappa-1}} u\left(\varepsilon^{-1} t, \varepsilon^{-\frac{m+2}{2}} x\right), \quad \varepsilon>0,
$$


also solves (1.1), with $u_{\varepsilon}(0, x)=\varphi_{\varepsilon}(x), \partial_{t} u_{\varepsilon}(0, x)=\psi_{\varepsilon}(x)$ for some resulting $\varphi_{\varepsilon}, \psi_{\varepsilon}$. Observe that

$$
\frac{\left\|\varphi_{\varepsilon}\right\|_{\dot{H}^{\gamma}\left(\mathbb{R}^{n}\right)}}{\|\varphi\|_{\dot{H}^{\gamma}\left(\mathbb{R}^{n}\right)}}=\frac{\left\|\psi_{\varepsilon}\right\|_{\dot{H}^{\gamma}\left(\mathbb{R}^{n}\right)}}{\|\psi\|_{\dot{H}^{\gamma}\left(\mathbb{R}^{n}\right)}}=\varepsilon^{\frac{m+2}{2}\left(\frac{n}{2}-\gamma\right)-\frac{2}{\kappa-1}},
$$

and $\frac{m+2}{2}\left(\frac{n}{2}-\gamma\right)-\frac{2}{\kappa-1}>0$ for $\gamma<\frac{n}{2}-\frac{4}{(m+2)(\kappa-1)}$. Hence, $\gamma<\frac{n}{2}-\frac{4}{(m+2)(\kappa-1)}$ implies that both the norm of the data $\left(\varphi_{\varepsilon}, \psi_{\varepsilon}\right)$ and the lifespan $T_{\varepsilon}=\varepsilon T$ of the solution $u_{\varepsilon}$ go to zero as $\varepsilon \rightarrow 0$, where $T$ is the lifespan of the solution $u$.

In case $\kappa_{*} \leq \kappa<\kappa_{2}$, as a supplement to Theorem 1.1, we consider the local existence and uniqueness of solutions $u$ of problem (1.1) in the space $C\left([0, T] ; \dot{H}^{\gamma}\left(\mathbb{R}^{n}\right)\right) \cap L^{s}\left((0, T) ; L^{q}\left(\mathbb{R}^{n}\right)\right)$ for certain $s \neq q$.

Theorem 1.4. Let $n \geq 2$, $F$ be above, $\gamma=\gamma(\kappa, m, n)$ be as in Theorem 1.1, and suppose that $\kappa_{*} \leq$ $\kappa<\kappa_{2}$. Then the unique solution $u$ of problem (1.1) also belongs to the space $L^{s}\left((0, T) ; L^{q}\left(\mathbb{R}^{n}\right)\right)$, where

$$
\frac{1}{q}=\frac{1}{(m+2)(n-1)}\left(\frac{8}{\kappa-1}-\frac{m}{\mu_{*}}\right)-\frac{n-1}{2(n+1)}
$$

and

$$
\frac{1}{s}=\frac{(m+2)(n-1)}{4}\left(\frac{1}{2}-\frac{1}{q}\right)+\frac{m}{4 \mu_{*}} .
$$

Moreover, estimate (1.4) is satisfied.

If $n \geq 3$ or $n=2, m \geq 3$, then we find a number $\gamma(\kappa, m, n)$ also for certain $\kappa$ in the range $1<\kappa<\kappa_{1}$.

Theorem 1.5. Let $n \geq 3$ or $n=2, m \geq 3$. Let $F$ be as above and $\kappa_{0} \leq \kappa<\kappa_{1}$. In addition, let the exponent $\gamma=\gamma(\kappa, m, n)$ in (1.1) be given by

$$
\gamma(\kappa, m, n)=\frac{n+1}{4}-\frac{n+1}{4 \mu_{*}(m+2)} \cdot \frac{\mu_{*}(m+2)(n-1)+12 \mu_{*}+2 m}{2 n \kappa-(n+1)}-\frac{m}{2 \mu_{*}(m+2)} .
$$

Then problem (1.1) possesses a unique solution $u \in C\left([0, T] ; \dot{H}^{\gamma}\left(\mathbb{R}^{n}\right)\right) \cap L^{s}\left((0, T) ; L^{q}\left(\mathbb{R}^{n}\right)\right)$ for some $T>0$, where

$$
\frac{1}{q}=\frac{1}{2 n \kappa-(n+1)}\left(\frac{n-1}{2}+\frac{6}{m+2}+\frac{m}{\mu_{*}(m+2)}\right)
$$

and

$$
\frac{1}{s}=\frac{(m+2)(n-1)}{4}\left(\frac{1}{2}-\frac{1}{q}\right)+\frac{m}{4 \mu_{*}} .
$$

Moreover, estimate (1.4) is satisfied.

Remark 1.6. Other than for the wave equation when $m=0$ (see also Remark 1.8 below), here $\gamma$ can be negative in certain situations. In fact, $\gamma(\kappa, m, n)<0$ holds in the following cases:

(i) $\kappa_{1}<\kappa<\frac{35}{17}\left(<\kappa_{*}\right)$ if $n=2, m=1$ and $\kappa_{1}<\kappa<\frac{13}{7}\left(<\kappa_{*}\right)$ if $n=2, m=2$ (see Theorem 1.1), 
(ii) $\kappa_{0}<\kappa<\frac{\mu_{*}\left(\mu_{*}+2\right)(n+1)}{\mu_{*}\left(\mu_{*}-1\right)(n+1)-m n}\left(\leq \kappa_{1}\right)$ if $n \geq 3$ or $n=2, m \geq 3$ (see Theorem 1.5).

Remark 1.7. For initial data $(\varphi, \psi)$ belonging to $H^{\gamma}\left(\mathbb{R}^{n}\right) \times H^{\gamma-\frac{2}{m+2}}\left(\mathbb{R}^{n}\right)$, where $\gamma \geq \gamma(\kappa, m, n)$, Theorems 1.1, 1.4, and 1.5 remain valid.

Remark 1.8. For $m=0,(1.1)$ becomes

$$
\left\{\begin{array}{l}
\partial_{t}^{2} u-\Delta u=F(u) \quad \text { in }(0, T) \times \mathbb{R}^{n}, \\
u(0, \cdot)=\varphi \in \dot{H}^{\gamma}\left(\mathbb{R}^{n}\right), \\
\partial_{t} u(0, \cdot)=\psi \in \dot{H}^{\gamma-1}\left(\mathbb{R}^{n}\right),
\end{array}\right.
$$

while the exponents $\kappa_{*}, \kappa_{0}, \kappa_{1}, \kappa_{2}$, and $\kappa_{3}$ are

$$
\begin{aligned}
& \kappa_{*}=\frac{n+3}{n-1}, \quad \kappa_{2}=\frac{(n+1)^{2}-6}{(n-1)^{2}-2}, \\
& \kappa_{1}=\frac{(n+1)^{2}}{(n-1)^{2}+4} \quad \text { if } n \geq 3, \\
& \kappa_{0}=1+\frac{3}{n}, \quad \kappa_{3}=\frac{n+1}{n-3} \quad \text { if } n \geq 4 .
\end{aligned}
$$

For $n \geq 3, \gamma$ defined in (1.3) equals

$$
\gamma(\kappa, 0, n)= \begin{cases}\frac{n+1}{4}-\frac{1}{\kappa-1} & \text { if } \kappa_{1}<\kappa \leq \kappa_{*}, \\ \frac{n}{2}-\frac{2}{\kappa-1} & \text { if } \kappa \geq \kappa_{*},\end{cases}
$$

whereas, for $n \geq 4, \gamma$ defined in (1.5) equals

$$
\gamma(\kappa, 0, n)=\frac{n+1}{4}-\frac{(n+1)(n+5)}{4} \frac{1}{2 n \kappa-(n+1)} .
$$

Note that the numbers in (1.6) and (1.7) are exactly those in (2.1) and (2.5) of [10]. In that paper, [10], the local existence problem for minimal regularity solutions of the semilinear wave equation was systematically studied. The results were achieved by establishing Strichartz-type estimates for the linear wave operator $\partial_{t}^{2}-\Delta$. Under certain restrictions on the nonlinearity $F(u, \nabla u)$, for the more general semilinear wave equation

$$
\left\{\begin{array}{l}
\partial_{t}^{2} u-\Delta u=F(u, \nabla u) \\
u(0, x)=\varphi(x), \quad \partial_{t} u(0, x)=\psi(x),
\end{array}\right.
$$

many remarkable results on the ill-posedness or well-posedness problem on the local existence of low regularity solutions have been obtained, see $[8-10,14,18,21]$ and the reference therein.

Remark 1.9. There are some essential differences between degenerate hyperbolic equations and strictly hyperbolic equations. Amongst others, the symmetry group is smaller (see [11]) and there is a loss of regularity for the linear Cauchy problem (see e.g. [4,22]). Therefore, as compared to the semilinear wave equation, a more delicate analysis is required when one studies minimal regularity results for the semilinear generalized Tricomi equation in the degenerate hyperbolic region. 
The Tricomi equation (i.e., Eq. (1.1) for $n=1, m=1$ ) were first studied by Tricomi [23] who initiated work on boundary value problems for linear partial differential operators of mixed elliptichyperbolic type. So far, these equations have been extensively studied in bounded domains under suitable boundary conditions and several applications to transonic flow problems were given (see $[3,6,13,23]$ and the references therein). Conservation laws for equations of mixed type were derived by Lupo and Payne [11,12]. In [17], we established the local solvability for low regularity solutions of the semilinear equation $\partial_{t}^{2} u-t^{m} \Delta u=F(u)$, where $n \geq 2, m \in \mathbb{N}$ is odd, in the domain $(-T, T) \times \mathbb{R}^{n}$ for some $T>0$. In $[1,24,26]$, fundamental solutions for the linear Tricomi operator and the linear generalized Tricomi operator have been explicitly computed. In case $n=2$ and $m=1$, Beals [2] obtained the local existence of the solution $u$ of the equation $\partial_{t}^{2} u-t \Delta u=F(u)$ with initial data of $H^{s}$-regularity, where $s>n / 2$. For the equation $\partial_{t}^{2} u-t^{m} \Delta u=a(t) F(u)$, where $n \geq 2$, $m \in \mathbb{N}$ is even, and both $a$ and $F$ are of power type, Yadgjian [25] obtained global existence and uniqueness for small data solutions provided the solution $v$ of the linear problem $\partial_{t}^{2} v-t^{m} \Delta v=0$ fulfills $t^{\beta} v \in C\left([0, \infty) ; L^{q}\left(\mathbb{R}^{n}\right)\right)$ for certain $\beta, q$ depending on $n, m$, and the powers occurring in $a$ and $F$. In $[15,16]$, for the semilinear generalized Tricomi equation $\partial_{t}^{2} u-t^{m} \Delta u=F(u)$ with initial data of a special structure, i.e., homogeneous of degree 0 or piecewise smooth along a hyperplane, we obtained local existence and uniqueness via establishing $L^{\infty}$ estimates on the solutions $v$ of the linear equation $\partial_{t}^{2} v-t^{m} \Delta v=g$. Note that when the nonlinear term $F(u)$ is of power type, for higher and higher powers of $\kappa$, these $L^{\infty}$ estimates are basically required to guarantee existence. In this paper, where the initial data in $\dot{H}^{\gamma}\left(\mathbb{R}^{n}\right)$ is of no special structure and $\gamma$ is minimal to guarantee local well-posedness of problem (1.1), the arguments of [15,16] fail. Inspired by the methods in [10], however, we are able to overcome the technical difficulties related to degeneracy and low regularity and eventually obtain the local well-posedness of problem (1.1).

We first study the linear problem

$$
\begin{cases}\partial_{t}^{2} u-t^{m} \Delta u=f(t, x) & \text { in }(0, T) \times \mathbb{R}^{n}, \\ u(0, \cdot)=\varphi(x), \quad \partial_{t} u(0, \cdot)=\psi(x) & \end{cases}
$$

and establish Strichartz-type estimates of the form

$$
\|u\|_{C_{t}^{0} \dot{H}_{x}^{\gamma}\left(S_{T}\right)}+\|u\|_{L_{t}^{s} L_{x}^{q}\left(S_{T}\right)} \leq C\left(\|\varphi\|_{\dot{H}^{\gamma}}+\|\psi\|_{\dot{H}^{\gamma-\frac{2}{m+2}}}+\|f\|_{L_{t}^{r} L_{x}^{p}\left(S_{T}\right)}\right)
$$

for certain $s, q, r, p$ (for details see below) and some constant $C=C(T, \gamma, s, q, r, p)>0$, where $S_{T}=(0, T) \times \mathbb{R}^{n}$. Note that, by scaling, a necessary condition for this estimate in case $T=\infty$ to hold is

$$
\frac{(m+2) n}{2}\left(\frac{1}{p}-\frac{1}{q}\right)+\frac{1}{r}-\frac{1}{s}=2 .
$$

In doing so, in Section 2, we introduce certain Fourier integral operators $W\left(=W^{0}\right)$ and $W^{\alpha}$ for $\alpha \in \mathbb{C}$. These operators depend on a parameter $\mu \geq 2$, introduced in (2.15), which plays an auxiliary role for the linear problems and agrees with the homogeneous dimension $\mu_{*}$ when applied to the semilinear problems. Along with the operators $W$ and $W^{\alpha}$ we also consider their parts $W_{j}$ and $W_{j}^{\alpha}$, respectively, resulting from a dyadic decomposition of frequency space. Continuity of the operators $W_{j}$ and $W_{j}^{\alpha}$ between function spaces which holds uniformly in $j$ ultimately provides linear estimates on the solutions $u$ of Eq. (1.8). 
In Section 3, we prove boundedness of the operators $W_{j}^{\alpha}$ from $L_{t}^{r} L_{x}^{p}\left(\mathbb{R}_{+}^{1+n}\right)$ to $L_{t}^{r^{\prime}} L_{x}^{p^{\prime}}\left(\mathbb{R}_{+}^{1+n}\right)$ (see Theorem 3.1) and from $L_{t}^{r} L_{x}^{p}\left(\mathbb{R}_{+}^{1+n}\right)$ to $L_{t}^{\infty} L_{x}^{2}\left(\mathbb{R}_{+}^{1+n}\right)$ (see Theorem 3.4), where $\mu$ has to satisfy the lower bound $\mu \geq \max \{2, m / 2\}$. Combining Theorem 3.1 and Stein's analytic interpolation theorem, we show boundedness of the operators $W_{j}^{\alpha}$ from $L^{q}\left(\mathbb{R}_{+}^{1+n}\right)$ to $L^{p_{0}}\left(\mathbb{R}_{+}^{1+n}\right)$, where $q_{0} \leq q \leq \infty$ (see Theorem 3.6). Through an additional dyadic decomposition now with respect to the time variable $t$, using Theorems 3.1 and 3.6 together with interpolation, we prove boundedness of the operators $W_{j}$ from $L_{t}^{r} L_{x}^{p}\left((0, T) \times \mathbb{R}^{n}\right)$ to $L_{t}^{s} L_{x}^{q}\left((0, T) \times \mathbb{R}^{n}\right)$ for any $T>0$ (see Theorems 3.7 and 3.8), where $\mu$ has to satisfy the new lower bounds $\mu \geq \mu_{*}$ (Theorem 3.7) and $\mu \geq \max \{2, m n / 2\}$ (Theorem 3.8), respectively.

In the sequel, we shall use the following notation:

$$
\frac{1}{p_{0}}=\frac{1}{2}+\frac{2 \mu-m}{\mu\left(2 \mu_{*}-m\right)}, \quad \frac{1}{p_{1}}=\frac{1}{2}+\frac{2 \mu-m}{\mu(m+2)(n-1)}, \quad \frac{1}{p_{2}}=\frac{2}{p_{0}}-\frac{1}{p_{1}} .
$$

Note that

$$
1<p_{1} \leq p_{0} \leq p_{2} \leq 2 \quad \text { if } n \geq 3 \text { or } n=2, m \geq 2,
$$

while $1 \leq p_{1}$ in case of $n=2$ and $m=1$ requires $\mu=2$ (and then $p_{1}=1$ ). For $1 \leq p \leq 2, p^{\prime}$ denotes the conjugate exponent of $p$ defined by $\frac{1}{p}+\frac{1}{p^{\prime}}=1$. Further, $q_{\ell}$ denotes $p_{\ell}^{\prime}$ for $\ell=0,1,2$, while $q_{0}^{*}$ equals $q_{0}$ when $\mu=\mu_{*}$ (see Remark 4.2). We often abbreviate function spaces $C_{t}^{0} \dot{H}_{x}^{\gamma}\left(S_{T}\right)=$ $C\left([0, T] ; \dot{H}^{\gamma}\left(\mathbb{R}^{n}\right)\right), L_{t}^{r} L_{x}^{p}\left(S_{T}\right)=L^{r}\left((0, T) ; L^{p}\left(\mathbb{R}^{n}\right)\right)$, and $A \lesssim B$ means that $A \leq C B$ holds for some generic constant $C>0$.

The paper is organized as follows: In Section 2, we define a class of Fourier integral operators associated with the linear generalized Tricomi operator $\partial_{t}^{2}-t^{m} \Delta$ in $\mathbb{R}_{+} \times \mathbb{R}^{n}$. Then, in Section 3 , we establish a series of mixed-norm space-time estimates for those Fourier integral operators. These estimates are applied, in Section 4, to obtain Strichartz-type estimates for the solutions of the linear generalized Tricomi equation which in turn, in Section 5, allow us to prove the local existence and uniqueness results for problem (1.1).

\section{SOME PRELIMINARIES}

In this section, we first recall an explicit formula for the solution of the linear generalized Tricomi equation obtained in [22] and then apply it to define a class of Fourier integral operators which will play a key role in proving our main results.

Consider the linear generalized Tricomi equation

$$
\left\{\begin{array}{l}
\partial_{t}^{2} u-t^{m} \Delta u=f(t, x) \quad \text { in } \mathbb{R}_{+} \times \mathbb{R}^{n} \\
u(0, \cdot)=\varphi, \quad \partial_{t} u(0, \cdot)=\psi
\end{array}\right.
$$

Its solution $u$ can be written as $u=v+w$, where $v$ solves the homogeneous equation

$$
\left\{\begin{array}{l}
\partial_{t}^{2} v-t^{m} \Delta v=0 \quad \text { in } \mathbb{R}_{+} \times \mathbb{R}^{n} \\
v(0, \cdot)=\varphi, \quad \partial_{t} v(0, \cdot)=\psi
\end{array}\right.
$$


and $w$ solves the inhomogeneous equation with zero initial data

$$
\left\{\begin{array}{l}
\partial_{t}^{2} w-t^{m} \Delta w=f(t, x) \text { in } \mathbb{R}_{+} \times \mathbb{R}^{n}, \\
w(0, \cdot)=\partial_{t} w(0, \cdot)=0 .
\end{array}\right.
$$

Recall that (see [22] or [25]) the solutions $v$ and $w$ of problems (2.2) and (2.3) can be expressed as

$$
v(t, x)=V_{0}\left(t, D_{x}\right) \varphi(x)+V_{1}\left(t, D_{x}\right) \psi(x)
$$

and

$$
w(t, x)=\int_{0}^{t}\left(V_{1}\left(t, D_{x}\right) V_{0}\left(\tau, D_{x}\right)-V_{0}\left(t, D_{x}\right) V_{1}\left(\tau, D_{x}\right)\right) f(\tau, x) d \tau,
$$

where the symbols $V_{j}(t, \xi)(j=0,1)$ of the Fourier integral operators $V_{j}\left(t, D_{x}\right)$ are

$$
\left\{\begin{array}{l}
V_{0}(t, \xi)=e^{-z / 2} \Phi\left(\frac{m}{2(m+2)}, \frac{m}{m+2} ; z\right), \\
V_{1}(t, \xi)=t e^{-z / 2} \Phi\left(\frac{m+4}{2(m+2)}, \frac{m+4}{m+2} ; z\right)
\end{array}\right.
$$

with $z=2 i \phi(t)|\xi|$ and $\phi(t)=(2 /(m+2)) t^{(m+2) / 2}$. Here, $\Phi(a, c ; z)$ is the confluent hypergeometric function which is an analytic function of $z$. Recall (see [5, page254]) that

$$
\frac{d^{n}}{d z^{n}} \Phi(a, c ; z)=\frac{(a)_{n}}{(c)_{n}} \Phi(a+n, c+n ; z),
$$

where $(a)_{0}=1,(a)_{n}=a(a+1) \ldots(a+n-1)$. In addition (see [25, (3.5)-(3.7)]), for $0<\arg (\mathrm{z})<\pi$, one has that

$$
e^{-z / 2} \Phi(a, c ; z)=\frac{\Gamma(c)}{\Gamma(a)} e^{z / 2} H_{+}(a, c ; z)+\frac{\Gamma(c)}{\Gamma(c-a)} e^{-z / 2} H_{-}(a, c ; z)
$$

where

$$
\begin{aligned}
& H_{+}(a, c ; z)=\frac{e^{-i \pi(c-a)}}{e^{i \pi(c-a)}-e^{-i \pi(c-a)}} \frac{1}{\Gamma(c-a)} z^{a-c} \int_{\infty}^{(0+)} e^{-\theta} \theta^{c-a-1}\left(1-\frac{\theta}{z}\right)^{a-1} d \theta \\
& H_{-}(a, c ; z)=\frac{1}{e^{i \pi a}-e^{-i \pi a}} \frac{1}{\Gamma(a)} z^{-a} \int_{\infty}^{(0+)} e^{-\theta} \theta^{a-1}\left(1+\frac{\theta}{z}\right)^{c-a-1} d \theta .
\end{aligned}
$$

Moreover, it holds that

$$
\begin{array}{ll}
\left|\partial_{\xi}^{\beta}\left(H_{+}(a, c ; 2 i \phi(t)|\xi|)\right)\right| \lesssim(\phi(t)|\xi|)^{a-c}(1+|\xi|)^{-|\beta|} & \text { if } \phi(t)|\xi| \geq 1 \\
\left|\partial_{\xi}^{\beta}\left(H_{-}(a, c ; 2 i \phi(t)|\xi|)\right)\right| \lesssim(\phi(t)|\xi|)^{-a}(1+|\xi|)^{-|\beta|} & \text { if } \phi(t)|\xi| \geq 1 .
\end{array}
$$

Choose $\eta \in C_{c}^{\infty}\left(\mathbb{R}_{+}\right)$such that $0 \leq \eta \leq 1$ with $\eta(r)=1$ if $r \leq 1$ and $\eta(r)=0$ if $r \geq 2$. Then from (2.5) and (2.7), we can write

$$
V_{0}\left(t, D_{x}\right) \varphi(x)=\int_{\mathbb{R}^{n}} e^{i(x \cdot \xi-\phi(t)|\xi|)} b_{1}(t, \xi) \hat{\varphi}(\xi) d \xi+\int_{\mathbb{R}^{n}} e^{i(x \cdot \xi+\phi(t)|\xi|)} b_{2}(t, \xi) \hat{\varphi}(\xi) d \xi
$$


and

$$
V_{1}\left(t, D_{x}\right) \psi(x)=\int_{\mathbb{R}^{n}} e^{i(x \cdot \xi-\phi(t)|\xi|)} b_{3}(t, \xi) \hat{\psi}(\xi) d \xi+\int_{\mathbb{R}^{n}} e^{i(x \cdot \xi+\phi(t)|\xi|)} b_{4}(t, \xi) \hat{\psi}(\xi) d \xi,
$$

where

$$
\begin{aligned}
& b_{1}(t, \xi)=\eta(\phi(t)|\xi|) \Phi\left(\frac{m}{2(m+2)}, \frac{m}{m+2} ; z\right)+(1-\eta(\phi(t)|\xi|)) H_{-}\left(\frac{m}{2(m+2)}, \frac{m}{m+2} ; z\right), \\
& b_{2}(t, \xi)=(1-\eta(\phi(t)|\xi|)) H_{+}\left(\frac{m}{2(m+2)}, \frac{m}{m+2} ; z\right),
\end{aligned}
$$

and

$$
\begin{aligned}
& b_{3}(t, \xi)=t \eta(\phi(t)|\xi|) \Phi\left(\frac{m+4}{2(m+2)}, \frac{m+4}{m+2} ; z\right)+t(1-\eta(\phi(t)|\xi|)) H_{-}\left(\frac{m+4}{2(m+2)}, \frac{m+4}{m+2} ; z\right), \\
& b_{4}(t, \xi)=t(1-\eta(\phi(t)|\xi|)) H_{+}\left(\frac{m+4}{2(m+2)}, \frac{m+4}{m+2} ; z\right) .
\end{aligned}
$$

We can also write

$$
\begin{aligned}
\int_{0}^{t} V_{0}\left(t, D_{x}\right) V_{1}\left(\tau, D_{x}\right) f(\tau, x) d \tau= & \int_{0}^{t} \int_{\mathbb{R}^{n}} e^{i(x \cdot \xi+(\phi(t)+\phi(\tau))|\xi|)} b_{2}(t, \xi) b_{4}(\tau, \xi) \hat{f}(\tau, \xi) d \xi d \tau \\
& +\int_{0}^{t} \int_{\mathbb{R}^{n}} e^{i(x \cdot \xi+(\phi(t)-\phi(\tau))|\xi|)} b_{2}(t, \xi) b_{3}(\tau, \xi) \hat{f}(\tau, \xi) d \xi d \tau \\
& +\int_{0}^{t} \int_{\mathbb{R}^{n}} e^{i(x \cdot \xi-(\phi(t)+\phi(\tau))|\xi|)} b_{1}(t, \xi) b_{3}(\tau, \xi) \hat{f}(\tau, \xi) d \xi d \tau \\
& +\int_{0}^{t} \int_{\mathbb{R}^{n}} e^{i(x \cdot \xi-(\phi(t)-\phi(\tau))|\xi|)} b_{1}(t, \xi) b_{4}(\tau, \xi) \hat{f}(\tau, \xi) d \xi d \tau
\end{aligned}
$$

and

$$
\begin{aligned}
\int_{0}^{t} V_{1}\left(t, D_{x}\right) V_{0}\left(\tau, D_{x}\right) f(\tau, x) d \tau= & \int_{0}^{t} \int_{\mathbb{R}^{n}} e^{i(x \cdot \xi+(\phi(t)+\phi(\tau))|\xi|)} b_{4}(t, \xi) b_{2}(\tau, \xi) \hat{f}(\tau, \xi) d \xi d \tau \\
& +\int_{0}^{t} \int_{\mathbb{R}^{n}} e^{i(x \cdot \xi-(\phi(t)-\phi(\tau))|\xi|)} b_{3}(t, \xi) b_{2}(\tau, \xi) \hat{f}(\tau, \xi) d \xi d \tau \\
& +\int_{0}^{t} \int_{\mathbb{R}^{n}} e^{i(x \cdot \xi-(\phi(t)+\phi(\tau))|\xi|)} b_{3}(t, \xi) b_{1}(\tau, \xi) \hat{f}(\tau, \xi) d \xi d \tau \\
& +\int_{0}^{t} \int_{\mathbb{R}^{n}} e^{i(x \cdot \xi+(\phi(t)-\phi(\tau))|\xi|)} b_{4}(t, \xi) b_{1}(\tau, \xi) \hat{f}(\tau, \xi) d \xi d \tau
\end{aligned}
$$

where $\hat{f}(\tau, \xi)$ is the Fourier transform of $f(\tau, x)$ with respect to the variable $x$ and $d \xi=(2 \pi)^{-n} d \xi$.

In view of the analyticity of $\Phi(a, c ; z)$ with respect to the variable $z$, identity (2.6), and estimates (2.8), we have that, for $(t, \xi) \in \mathbb{R}_{+}^{1+n}$,

$$
\left|\partial_{\xi}^{\beta} b_{\ell}(t, \xi)\right| \lesssim(1+\phi(t)|\xi|)^{-\frac{m}{2(m+2)}}|\xi|^{-|\beta|}, \quad \ell=1,2
$$


and

$$
\left|\partial_{\xi}^{\beta} b_{\ell}(t, \xi)\right| \lesssim t(1+\phi(t)|\xi|)^{-\frac{m+4}{2(m+2)}}|\xi|^{-|\beta|}, \quad \ell=3,4
$$

Thus, for $\ell=1,2, k=3,4, \mu \geq 2, t, \tau>0$, and $\xi \in \mathbb{R}^{n}$, one has from (2.13) and (2.14) that

$$
\begin{aligned}
\left|\partial_{\xi}^{\beta}\left(b_{k}(t, \xi) b_{\ell}(\tau, \xi)\right)\right| & \lesssim t(1+\phi(t)|\xi|)^{-\frac{m+4}{2(m+2)}}(1+\phi(\tau)|\xi|)^{-\frac{m}{2(m+2)}}|\xi|^{-|\beta|} \\
& \lesssim(1+\phi(t)|\xi|)^{-\frac{m}{2(m+2)}}(1+\phi(\tau)|\xi|)^{-\frac{m}{2(m+2)}}|\xi|^{-\frac{2}{m+2}}-|\beta| \\
& \lesssim(1+|\phi(t)-\phi(\tau)||\xi|)^{-\frac{m}{\mu(m+2)}}|\xi|^{-\frac{2}{m+2}-|\beta|} .
\end{aligned}
$$

Furthermore, estimates (2.13)-(2.15) yield that, for $\ell=1,2, k=3,4$ or $\ell=3,4, k=1,2$ and for $\mu \geq 2, t, s>0$, and $\xi \in \mathbb{R}^{n}$, one has

$$
\begin{aligned}
\left|\partial_{\xi}^{\beta}\left(\int_{t}^{\infty} \overline{b_{\ell}(\tau, \xi) b_{k}(t, \xi)} \partial_{\tau}\left(b_{\ell}(\tau, \xi) b_{k}(s, \xi)\right) d \tau\right)\right| & \\
& \lesssim(1+|\phi(t)-\phi(s)||\xi|)^{-\frac{m}{\mu(m+2)}}|\xi|^{-\frac{4}{m+2}-|\beta|}
\end{aligned}
$$

and

$$
\begin{aligned}
\left|\partial_{\xi}^{\beta}\left(\int_{s}^{\infty} \overline{b_{\ell}(\tau, \xi) b_{k}(t, \xi)} \partial_{\tau}\left(b_{\ell}(\tau, \xi) b_{k}(s, \xi)\right) d \tau\right)\right| & \\
& \lesssim(1+|\phi(t)-\phi(s)||\xi|)^{-\frac{m}{\mu(m+2)}}|\xi|^{-\frac{4}{m+2}-|\beta|}
\end{aligned}
$$

In order to study the function $w$ in (2.4), in view of (2.11), (2.12) and (2.15)-(2.17), it suffices to consider, for a given $\mu \geq 2$, the Fourier integral operator $W$,

$$
W f(t, x)=\int_{0}^{t} \int_{\mathbb{R}^{n}} e^{i(x \cdot \xi+(\phi(t)-\phi(s))|\xi|)} b(t, s, \xi) \hat{f}(s, \xi) d \xi d s,
$$

where $b \in C^{\infty}\left(\mathbb{R}_{+} \times \mathbb{R}_{+} \times \mathbb{R}^{n}\right)$ satisfies

(i) for $t, s>0$ and $\xi \in \mathbb{R}^{n}$,

$$
\left|\partial_{\xi}^{\beta} b(t, s, \xi)\right| \lesssim(1+|\phi(t)-\phi(s)||\xi|)^{-\frac{m}{\mu(m+2)}}|\xi|^{-\frac{2}{m+2}-|\beta|}
$$

(ii) for $t, s>0$ and $\xi \in \mathbb{R}^{n}$,

$$
\left|\partial_{\xi}^{\beta}\left(\int_{t}^{\infty} \overline{b(\tau, t, \xi)} \partial_{\tau} b(\tau, s, \xi) d \tau\right)\right| \lesssim(1+|\phi(t)-\phi(s)||\xi|)^{-\frac{m}{\mu(m+2)}}|\xi|^{-\frac{4}{m+2}-|\beta|}
$$

and

$$
\left|\partial_{\xi}^{\beta}\left(\int_{s}^{\infty} \overline{b(\tau, t, \xi)} \partial_{\tau} b(\tau, s, \xi) d \tau\right)\right| \lesssim(1+|\phi(t)-\phi(s)||\xi|)^{-\frac{m}{\mu(m+2)}}|\xi|^{-\frac{4}{m+2}-|\beta|} .
$$

Let $\Theta \in C_{c}^{\infty}\left(\mathbb{R}_{+}\right)$satisfy supp $\Theta \subseteq[1 / 2,2]$ and

$$
\sum_{j=-\infty}^{\infty} \Theta\left(t / 2^{j}\right)=1 \text { for } t>0
$$

Then, as in [10], for $j \in \mathbb{Z}$ and $\alpha \in \mathbb{C}$, we define dyadic operators $W_{j}$ and $W_{j}^{\alpha}$,

$$
W_{j} f(t, x)=\int_{0}^{t} \int_{\mathbb{R}^{n}} e^{i(x \cdot \xi+(\phi(t)-\phi(s))|\xi|)} b_{j}(t, s, \xi) \hat{f}(s, \xi) d \xi d s
$$


and

$$
W_{j}^{\alpha} f(t, x)=\int_{0}^{t} \int_{\mathbb{R}^{n}} e^{i(x \cdot \xi+(\phi(t)-\phi(s))|\xi|)} b_{j}(t, s, \xi) \hat{f}(s, \xi) \frac{d \xi}{|\xi|^{\alpha}} d s,
$$

where $b_{j}(t, s, \xi)=\Theta\left(|\xi| / 2^{j}\right) b(t, s, \xi)$; here $b \in C^{\infty}\left(\mathbb{R}_{+} \times \mathbb{R}_{+} \times \mathbb{R}^{n}\right)$ satisfies the estimates (2.19)(2.21).

Littlewood-Paley theory gives us a relationship between $W f$ and $W_{j} f\left(=W_{j}^{0} f\right)$, which will play an important role in our arguments in Section 4.

Proposition 2.1. Let $n \geq 2$. For $1<p \leq 2,1 \leq r \leq 2,2 \leq q<\infty$, and $2 \leq s \leq \infty$, let

$$
\left\|W_{j} f\right\|_{L_{t}^{s} L_{x}^{q}} \lesssim\|f\|_{L_{t}^{r} L_{x}^{p}}
$$

hold uniformly in $j$. Then

$$
\|W f\|_{L_{t}^{s} L_{x}^{q}} \lesssim\|f\|_{L_{t}^{r} L_{x}^{p}}
$$

Proof. This is actually an application of Lemma 3.8 of [10]. For the sake of completeness, we give the proof here. By Littlewood-Paley theory (see, e.g., [20]), for any $1<\rho<\infty$,

$$
\|W f(t, \cdot)\|_{L^{\rho}\left(\mathbb{R}^{n}\right)} \lesssim\left\|\left(\sum_{j=-\infty}^{\infty}\left|W_{j} f(t, \cdot)\right|^{2}\right)^{1 / 2}\right\|_{L^{\rho}\left(\mathbb{R}^{n}\right)} \lesssim\|W f(t, \cdot)\|_{L^{\rho}\left(\mathbb{R}^{n}\right)} .
$$

Together with the Minkowski inequality, this yields

$$
\|W f\|_{L_{t}^{s} L_{x}^{q}} \lesssim\left(\sum_{j=-\infty}^{\infty}\left\|W_{j} f\right\|_{L_{t}^{s} L_{x}^{q}}^{2}\right)^{1 / 2}
$$

and

$$
\left(\sum_{j=-\infty}^{\infty}\left\|W_{j} f\right\|_{L_{t}^{r} L_{x}^{p}}^{2}\right)^{1 / 2} \lesssim\|W f\|_{L_{t}^{r} L_{x}^{p}}
$$

Notice that

$$
f=\sum_{k=-\infty}^{\infty} f_{k}
$$

where $f_{k}(\tau, x)=\Theta\left(\tau / 2^{k}\right) f(\tau, x)$. Therefore, for some $M_{0} \in \mathbb{N}$,

$$
\begin{aligned}
\|W f\|_{L_{t}^{s} L_{x}^{q}}^{2} & \lesssim \sum_{j=-\infty}^{\infty}\left\|W_{j} f\right\|_{L_{t}^{s} L_{x}^{q}}^{2} & & \text { (by (2.24)) } \\
& =\sum_{j=-\infty}^{\infty}\left\|W_{j}\left(\sum_{|j-k| \leq M_{0}} f_{k}\right)\right\|_{L_{t}^{s} L_{x}^{q}}^{2} & & \text { (due to the compact support of } \Theta \text { ) } \\
& \lesssim \sum_{j=-\infty}^{\infty}\left(\sum_{|j-k| \leq M_{0}}\left\|W_{j} f_{k}\right\|_{L_{t}^{s} L_{x}^{q}}\right)^{2} & & \text { (by Minkowski inequality) } \\
& \lesssim \sum_{j=-\infty}^{\infty} \sum_{|j-k| \leq M_{0}}\left\|f_{k}\right\|_{L_{t}^{r} L_{x}^{p}}^{2} & & \text { (by (2.23)) }
\end{aligned}
$$




$$
\left.\lesssim \sum_{j=-\infty}^{\infty}\left\|f_{j}\right\|_{L_{t}^{r} L_{x}^{p}}^{2} \lesssim\|f\|_{L_{t}^{r} L_{x}^{p}}^{2} \quad \quad \text { (by }(2.25)\right)
$$

which completes the proof of Proposition 2.1.

\section{MIXED-NORM ESTIMATES FOR A CLASS OF FOURIER INTEGRAL OPERATORS}

In this section, for $j \in \mathbb{Z}, \alpha \in \mathbb{C}$, and $\mu \geq 2$, we shall study mixed norm estimates for the class of Fourier integral operators $W_{j}^{\alpha}$ defined in (2.22).

We start by considering the boundedness of the operator $W_{j}^{\alpha}$ from $L_{t}^{r} L_{x}^{p}$ to $L_{t}^{r^{\prime}} L_{x}^{p^{\prime}}$, where $1<$ $r, p \leq 2$. We denote $\lambda_{j}=2^{j}$. All the following estimates hold uniformly in $j$.

Theorem 3.1. Let $n \geq 2$ and $\mu \geq \max \{2, m / 2\}$. Then:

(i) For $\max \left\{p_{1}, 1\right\}<p \leq 2$ and

$$
\frac{1}{r}=1-\frac{m}{4 \mu}-\frac{(m+2)(n-1)}{4}\left(\frac{1}{p}-\frac{1}{2}\right),
$$

we have that

$$
\left\|W_{j}^{\alpha} f\right\|_{L_{t}^{r^{\prime}} L_{x}^{p^{\prime}}\left(\mathbb{R}_{+}^{1+n}\right)} \lesssim \lambda_{j}^{\left(\frac{1}{p}-\frac{1}{2}\right)(n+1)-\frac{m}{\mu(m+2)}-\frac{2}{m+2}-\operatorname{Re} \alpha}\|f\|_{L_{t}^{r} L_{x}^{p}\left(\mathbb{R}_{+}^{1+n}\right)}
$$

Consequently,

$$
\left\|W_{j}^{\alpha} f\right\|_{L_{t}^{r^{\prime}} L_{x}^{p^{\prime}}\left(\mathbb{R}_{+}^{1+n}\right)} \lesssim\|f\|_{L_{t}^{r} L_{x}^{p}\left(\mathbb{R}_{+}^{1+n}\right)} \quad \text { if } \operatorname{Re} \alpha=\left(\frac{1}{p}-\frac{1}{2}\right)(n+1)-\frac{m}{\mu(m+2)}-\frac{2}{m+2} .
$$

(ii) For $p_{1}>1$ and $1<p<p_{1}$, we have that

$$
\left\|W_{j}^{\alpha} f\right\|_{L_{t}^{2} L_{x}^{p^{\prime}\left(\mathbb{R}_{+}^{1+n}\right)}} \lesssim \lambda_{j}^{n\left(\frac{2}{p}-1\right)-\frac{4}{m+2}-\operatorname{Re} \alpha}\|f\|_{L_{t}^{2} L_{x}^{p}\left(\mathbb{R}_{+}^{1+n}\right)} .
$$

In particular,

$$
\left\|W_{j}^{\alpha} f\right\|_{L_{t}^{2} L_{x}^{p^{\prime}}\left(\mathbb{R}_{+}^{1+n}\right)} \lesssim\|f\|_{L_{t}^{2} L_{x}^{p}\left(\mathbb{R}_{+}^{1+n}\right)} \quad \text { if } \operatorname{Re} \alpha=n\left(\frac{2}{p}-1\right)-\frac{4}{m+2} .
$$

To prove Theorem 3.1, for fixed $t, \tau>0$, we first consider the operator $B_{j}^{\alpha}$,

$$
B_{j}^{\alpha} f(t, \tau, x)=\int_{\mathbb{R}^{n}} e^{i(x \cdot \xi+(\phi(t)-\phi(\tau))|\xi|)} b_{j}(t, \tau, \xi) \hat{f}(\tau, \xi) \frac{d \xi}{|\xi|^{\alpha}} .
$$

Lemma 3.2. Let $n \geq 2$ and $1 \leq p \leq 2$. Then, for $t, \tau>0$,

$$
\begin{aligned}
\left\|B_{j}^{\alpha} f(t, \tau, \cdot)\right\|_{L^{p^{\prime}}\left(\mathbb{R}^{n}\right)} \lesssim \lambda_{j}^{\left(\frac{1}{p}-\frac{1}{2}\right)(n+1)-\frac{m}{\mu(m+2)}-\frac{2}{m+2}-\operatorname{Re} \alpha} \\
\quad \times\left(\lambda_{j}^{-\frac{2}{m+2}}+|t-\tau|\right)^{-(m+2)\left(\frac{1}{p}-\frac{1}{2}\right) \frac{n-1}{2}-\frac{m}{2 \mu}}\|f(\tau, \cdot)\|_{L^{p}\left(\mathbb{R}^{n}\right)} .
\end{aligned}
$$

Proof. Denote

$$
K_{j}^{\alpha}(t, \tau, x, y)=\int_{\mathbb{R}^{n}} e^{i((x-y) \cdot \xi+(\phi(t)-\phi(\tau))|\xi|)} b_{j}(t, \tau, \xi) \frac{d \xi}{|\xi|^{\alpha}}
$$


Then $B_{j}^{\alpha} f$ can be written as

$$
B_{j}^{\alpha} f(t, \tau, x)=\int_{\mathbb{R}^{n}} K_{j}^{\alpha}(t, \tau, x, y) f(\tau, y) d y .
$$

Since $\operatorname{supp}_{\xi} b_{j} \subseteq\left\{\xi \in \mathbb{R}^{n}\left|\lambda_{j} / 2 \leq\right| \xi \mid \leq 2 \lambda_{j}\right\}$, we have from (2.19) that

$$
\left|\partial_{\xi}^{\beta} b_{j}(t, \tau, \xi)\right| \lesssim \lambda_{j}^{-\frac{m}{\mu(m+2)}-\frac{2}{m+2}-|\beta|}\left(\lambda_{j}^{-\frac{2}{m+2}}+|t-\tau|\right)^{-\frac{m}{2 \mu}} .
$$

We now apply (3.8) to derive estimate (3.6) by Plancherel's theorem when $p=2$ and by the stationary phase method when $p=1$. By interpolation, we then obtain (3.6) for $1<p<2$.

Indeed, it follows from Plancherel's theorem that

$$
\begin{aligned}
\left\|B_{j}^{\alpha} f(t, \tau, \cdot)\right\|_{L_{x}^{2}\left(\mathbb{R}^{n}\right)} & =\left\|e^{i(\phi(t)-\phi(\tau))|\xi|} b_{j}(t, \tau, \xi) \hat{f}(\tau, \xi)|\xi|^{-\alpha}\right\|_{L_{\xi}^{2}\left(\mathbb{R}^{n}\right)} \\
& \lesssim \lambda_{j}^{-\frac{m}{\mu(m+2)}-\frac{2}{m+2}-\operatorname{Re} \alpha}\left(\lambda_{j}^{-\frac{2}{m+2}}+|t-\tau|\right)^{-\frac{m}{2 \mu}}\|f(\tau, \cdot)\|_{L^{2}\left(\mathbb{R}^{n}\right)} .
\end{aligned}
$$

On the other hand, by the stationary phase method (see e.g. [19, Lemma 7.2.4]), we have that, for any $N \geq 0$,

$$
\begin{aligned}
&\left|K_{j}^{\alpha}(t, \tau, x, y)\right| \lesssim \lambda_{j}^{n}(1\left.+|\phi(t)-\phi(\tau)| \lambda_{j}\right)^{-\frac{n-1}{2}}\left(\lambda_{j}^{-\frac{2}{m+2}}+|t-\tau|\right)^{-\frac{m}{2 \mu}} \\
& \times \lambda_{j}^{-\frac{m}{\mu(m+2)}-\frac{2}{m+2}-\operatorname{Re} \alpha}\left(1+\lambda_{j}|| x-y|-| \phi(t)-\phi(\tau)||\right)^{-N} \\
& \lesssim \lambda_{j}^{\frac{n+1}{2}-\frac{m}{\mu(m+2)}-\frac{2}{m+2}-\operatorname{Re} \alpha}\left(\lambda_{j}^{-\frac{2}{m+2}}+|t-\tau|\right)^{-\frac{(m+2)(n-1)}{4}-\frac{m}{2 \mu}} \\
& \quad \times\left(1+\lambda_{j}|| x-y|-| \phi(t)-\phi(\tau)||\right)^{-N} .
\end{aligned}
$$

Choosing $N=0$ in (3.10) gives

$$
\begin{aligned}
& \left\|\left(B_{j}^{\alpha} f\right)(t, \tau, \cdot)\right\|_{L^{\infty}\left(\mathbb{R}^{n}\right)} \leq\left\|K_{j}^{\alpha}(t, \tau, \cdot, \cdot)\right\|_{L_{x, y}^{\infty}}\|f(\tau, \cdot)\|_{L^{1}\left(\mathbb{R}^{n}\right)} \\
& \quad \lesssim \lambda_{j}^{\frac{n+1}{2}-\frac{m}{\mu(m+2)}-\frac{2}{m+2}-\operatorname{Re} \alpha}\left(\lambda_{j}^{-\frac{2}{m+2}}+|t-\tau|\right)^{-\frac{(m+2)(n-1)}{4}-\frac{m}{2 \mu}}\|f(\tau, \cdot)\|_{L^{1}\left(\mathbb{R}^{n}\right)}
\end{aligned}
$$

Interpolation between (3.9) and (3.11) yields (3.6) in case $1 \leq p \leq 2$ which completes the proof of estimate (3.6).

Proof of Theorem 3.1. Now we return to the proof of Theorem 3.1. From (3.7), we have

$$
W_{j}^{\alpha} f(t, x)=\int_{0}^{t}\left(B_{j}^{\alpha} f\right)(t, \tau, x) d \tau .
$$

Using Minkowski's inequality and estimate (3.6), we thus have that

$$
\begin{aligned}
& \left\|W_{j}^{\alpha} f(t, \cdot)\right\|_{L^{p^{\prime}\left(\mathbb{R}^{n}\right)}} \lesssim \lambda_{j}^{\left(\frac{1}{p}-\frac{1}{2}\right)(n+1)-\frac{m}{\mu(m+2)}-\frac{2}{m+2}-\operatorname{Re} \alpha} \\
& \times \int_{0}^{\infty}\left(\lambda_{j}^{-\frac{2}{m+2}}+|t-\tau|\right)^{-(m+2)\left(\frac{1}{p}-\frac{1}{2}\right) \frac{n-1}{2}-\frac{m}{2 \mu}}\|f(\tau, \cdot)\|_{L^{p}\left(\mathbb{R}^{n}\right)} d \tau .
\end{aligned}
$$


1) Case $\max \left\{\boldsymbol{p}_{1}, \mathbf{1}\right\}<\boldsymbol{p} \leq \mathbf{2}$. In this case, we have $1<r<2$. Note that

$$
\frac{1}{r}-\frac{1}{r^{\prime}}=-(m+2)\left(\frac{1}{p}-\frac{1}{2}\right) \frac{n-1}{2}-\frac{m}{2 \mu}+1 \text {. }
$$

Then it follows from the Hardy-Littlewood-Sobolev theorem and (3.13) that estimate (3.2) holds.

2) Case $p_{1}>1$ and $1<p<p_{1}$. In this case,

$$
(m+2)\left(\frac{1}{p}-\frac{1}{2}\right) \frac{n-1}{2}+\frac{m}{2 \mu}>1 .
$$

Thus,

$$
\sup _{t>0} \int_{0}^{\infty}\left(\lambda_{j}^{-\frac{2}{m+2}}+|t-\tau|\right)^{-(m+2)\left(\frac{1}{p}-\frac{1}{2}\right) \frac{n-1}{2}-\frac{m}{2 \mu}} d \tau<\infty
$$

which together with Schur's lemma and (3.13) yields (3.4).

We would like to stress that in the proof of Theorem 3.1 only condition (2.19) on the function $b \in C^{\infty}\left(\mathbb{R}_{+} \times \mathbb{R}_{+} \times \mathbb{R}^{n}\right)$ was used, whereas the conditions (2.20) and (2.21) were not required,

Remark 3.3. Note that the adjoint operator $\left(W_{j}^{\alpha}\right)^{*}$ of $W_{j}^{\alpha}$ is of the form

$$
\left(W_{j}^{\alpha}\right)^{*} f(t, x)=\int_{t}^{\infty} \int_{\mathbb{R}^{n}} e^{i(x \cdot \xi+(\phi(t)-\phi(\tau))|\xi|)} \overline{b_{j}(\tau, t, \xi)} \hat{f}(\tau, \xi) \frac{d \xi}{|\xi|^{\alpha}} d \tau .
$$

By duality, we infer from Theorem 3.1 that

$$
\left\|\left(W_{j}^{\alpha}\right)^{*} f\right\|_{L_{t}^{r^{\prime}} L_{x}^{p^{\prime}}\left(\mathbb{R}_{+}^{1+n}\right)} \lesssim \lambda_{j}^{\left(\frac{1}{p}-\frac{1}{2}\right)(n+1)-\frac{m}{\mu(m+2)}-\frac{2}{m+2}-\operatorname{Re} \alpha}\|f\|_{L_{t}^{r} L_{x}^{p}\left(\mathbb{R}_{+}^{1+n}\right)}
$$

if $\max \left\{p_{1}, 1\right\}<p \leq 2$ and

$$
\left\|\left(W_{j}^{\alpha} f\right)^{*}\right\|_{L_{t}^{2} L_{x}^{p^{\prime}}\left(\mathbb{R}_{+}^{1+n}\right)} \lesssim \lambda_{j}^{n\left(\frac{2}{p}-1\right)-\frac{4}{m+2}-\operatorname{Re} \alpha}\|f\|_{L_{t}^{2} L_{x}^{p}\left(\mathbb{R}_{+}^{1+n}\right)} .
$$

if $p_{1}>1$ and $1<p<p_{1}$. Here, $r$ is given in (3.1).

As an application of Theorem 3.1, we obtain the boundedness of the operator $W_{j}^{\alpha}$ from $L_{t}^{r} L_{x}^{p}$ to $L_{t}^{\infty} L_{x}^{2}$, where $1<r, p \leq 2$.

Theorem 3.4. Let $n \geq 2$ and $\mu \geq \max \{2, m / 2\}$. Then:

(i) For $\max \left\{p_{1}, 1\right\}<p \leq 2$ and $r$ be as in (3.1), we have that

$$
\left\|W_{j}^{\alpha} f\right\|_{L_{t}^{\infty} L_{x}^{2}\left(\mathbb{R}_{+}^{1+n}\right)} \lesssim \lambda_{j}^{\left(\frac{1}{p}-\frac{1}{2}\right) \frac{n+1}{2}-\frac{m}{2 \mu(m+2)}-\frac{2}{m+2}-\operatorname{Re} \alpha}\|f\|_{L_{t}^{r} L_{x}^{p}\left(\mathbb{R}_{+}^{1+n}\right)} .
$$

Consequently,

$$
\left\|W_{j}^{\alpha} f\right\|_{L_{t}^{\infty} L_{x}^{2}\left(\mathbb{R}_{+}^{1+n}\right)} \lesssim\|f\|_{L_{t}^{r} L_{x}^{p}\left(\mathbb{R}_{+}^{1+n}\right)} \quad \text { if } \operatorname{Re} \alpha=\left(\frac{1}{p}-\frac{1}{2}\right) \frac{n+1}{2}-\frac{m}{2 \mu(m+2)}-\frac{2}{m+2} \text {. }
$$

(ii) For $p_{1}>1$ and $1<p<p_{1}$, we have that

$$
\left\|W_{j}^{\alpha} f\right\|_{L_{t}^{\infty} L_{x}^{2}\left(\mathbb{R}_{+}^{1+n}\right)} \lesssim \lambda_{j}^{n\left(\frac{1}{p}-\frac{1}{2}\right)-\frac{3}{m+2}-\operatorname{Re} \alpha}\|f\|_{L_{t}^{2} L_{x}^{p}\left(\mathbb{R}_{+}^{1+n}\right)} .
$$


In particular,

$$
\left\|W_{j}^{\alpha} f\right\|_{L_{t}^{\infty} L_{x}^{2}\left(\mathbb{R}_{+}^{1+n}\right)} \lesssim\|f\|_{L_{t}^{2} L_{x}^{p}\left(\mathbb{R}_{+}^{1+n}\right)} \quad \text { if } \operatorname{Re} \alpha=n\left(\frac{1}{p}-\frac{1}{2}\right)-\frac{3}{m+2} .
$$

Proof. For given $j \in \mathbb{Z}$ and $\alpha \in \mathbb{C}$, denote $U=W_{j}^{\alpha} f$. Then from (2.22) we have

$$
U(t)=\int_{0}^{t} e^{i(\phi(t)-\phi(\tau)) \sqrt{-\Delta}} b_{j}\left(t, \tau, D_{x}\right)(-\Delta)^{-\alpha / 2} f(\tau) d \tau,
$$

where $b_{j}\left(t, \tau, D_{x}\right)$ is the pseudodifferential operator with full symbol $b_{j}(t, \tau, \xi)$. Then $U(t)$ solves the Cauchy problem

$$
\left\{\begin{array}{l}
i \partial_{t} U(t)=-t^{m / 2} \sqrt{-\Delta} U(t)+i b_{j}\left(t, t, D_{x}\right)(-\Delta)^{-\alpha / 2} f(t) \\
\quad+i \int_{0}^{t} e^{i(\phi(t)-\phi(\tau)) \sqrt{-\Delta}} \partial_{t} b_{j}\left(t, \tau, D_{x}\right)(-\Delta)^{-\alpha / 2} f(\tau) d \tau \\
U(0)=0 .
\end{array}\right.
$$

Multiplying by $\overline{U(t)}$ and then integrating over $\mathbb{R}^{n}$ yields

$$
\begin{aligned}
i\left\langle\partial_{t} U(t), U(t)\right\rangle=-t^{m / 2}\langle & \sqrt{-\Delta} U(t), U(t)\rangle+i\left\langle b_{j}\left(t, t, D_{x}\right)(-\Delta)^{-\alpha / 2} f(t), U(t)\right\rangle \\
& +i\left\langle\int_{0}^{t} e^{i(\phi(t)-\phi(\tau)) \sqrt{-\Delta}} \partial_{t} b_{j}\left(t, \tau, D_{x}\right)(-\Delta)^{-\alpha / 2} f(\tau) d \tau, U(t)\right\rangle,
\end{aligned}
$$

and, therefore,

$$
\begin{aligned}
\frac{1}{2} \frac{d}{d t}\|U(t)\|^{2}=\operatorname{Re}\left\langle\int_{0}^{t} e^{i(\phi(t)-\phi(\tau)) \sqrt{-\Delta}} \partial_{t} b_{j}\left(t, \tau, D_{x}\right)(-\Delta)^{-\alpha / 2} f(\tau) d \tau, U(t)\right\rangle \\
+\operatorname{Re}\left\langle b_{j}^{*}\left(t, t, D_{x}\right)(-\Delta)^{-\alpha / 2} U(t), f(t)\right\rangle .
\end{aligned}
$$

Consequently,

$$
\begin{aligned}
\|U(s)\|^{2}= & 2 \operatorname{Re} \int_{0}^{s}\left\langle\int_{0}^{t} e^{i(\phi(t)-\phi(\tau)) \sqrt{-\Delta}} \partial_{t} b_{j}\left(t, \tau, D_{x}\right)(-\Delta)^{-\alpha / 2} f(\tau) d \tau, U(t)\right\rangle d t \\
& +2 \operatorname{Re} \int_{0}^{s}\left\langle b_{j}^{*}\left(t, t, D_{x}\right)(-\Delta)^{-\alpha / 2} U(t), f(t)\right\rangle d t \\
\lesssim & \left|\int_{0}^{s} \int_{\mathbb{R}^{n}} L_{j}^{\alpha} f(t, x) \overline{W_{j}^{\alpha} f(t, x)} d x d t\right| \\
& \quad+\left|\int_{0}^{s} \int_{\mathbb{R}^{n}} b_{j}^{*}\left(t, t, D_{x}\right) W_{j}^{2 \alpha} f(t, x) \overline{f(t, x)} d x d t\right| \\
= & \mathrm{I}+\mathrm{II},
\end{aligned}
$$

where

$$
\begin{aligned}
\mathrm{I} & =\left|\int_{0}^{s} \int_{\mathbb{R}^{n}} L_{j}^{\alpha} f(t, x) \overline{W_{j}^{\alpha} f(t, x)} d x d t\right| \\
\mathrm{II} & =\left|\int_{0}^{s} \int_{\mathbb{R}^{n}} b_{j}^{*}\left(t, t, D_{x}\right) W_{j}^{2 \alpha} f(t, x) \overline{f(t, x)} d x d t\right|,
\end{aligned}
$$


and

$$
L_{j}^{\alpha} f(t, x)=\int_{0}^{t} \int_{\mathbb{R}^{n}} e^{i(x \cdot \xi+(\phi(t)-\phi(\tau))|\xi|)} \partial_{t} b_{j}(t, \tau, \xi) \hat{f}(\tau, \xi) \frac{d \xi}{|\xi|^{\alpha}} d \tau .
$$

From (2.19), one has that, for any fixed $t>0, b_{j}\left(t, t, D_{x}\right) \in \Psi^{-\frac{2}{m+2}}\left(\mathbb{R}^{n}\right)$, and then $b_{j}^{*}\left(t, t, D_{x}\right) \in$ $\Psi^{-\frac{2}{m+2}}\left(\mathbb{R}^{n}\right)$, which yields that the term II is essentially

$$
\left|\int_{0}^{s} \int_{\mathbb{R}^{n}}\left(W_{j}^{2 \alpha+\frac{2}{m+2}} f\right)(t, x) \overline{f(t, x)} d x d t\right|
$$

and thus by application of Theorem 3.1 it follows that

$$
\mathrm{II} \lesssim \begin{cases}\lambda_{j}^{(n+1)\left(\frac{1}{p}-\frac{1}{2}\right)-\frac{m}{\mu(m+2)}-\frac{4}{m+2}-2 \operatorname{Re} \alpha}\|f\|_{L_{t}^{r} L_{x}^{p}\left(\mathbb{R}_{+}^{1+n}\right)}^{2} & \text { if } \max \left\{p_{1}, 1\right\}<p \leq 2, \\ \lambda_{j}^{n\left(\frac{2}{p}-1\right)-\frac{6}{m+2}-2 \operatorname{Re} \alpha}\|f\|_{L_{t}^{2} L_{x}^{p}\left(\mathbb{R}_{+}^{1+n}\right)}^{2} & \text { if } p_{1}>1 \text { and } 1<p<p_{1} .\end{cases}
$$

As for the term I, note that

$$
\mathrm{I}=\left|\int_{0}^{s} \int_{\mathbb{R}^{n}}\left(W_{j}^{\alpha}\right)^{*} L_{j}^{\alpha} f(t, x) \overline{f(t, x)} d x d t\right| \leq\left\|\left(W_{j}^{\alpha}\right)^{*} L_{j}^{\alpha} f\right\|_{L_{t}^{\rho^{\prime}} L_{x}^{p^{\prime}}\left(\mathbb{R}_{+}^{1+n}\right)}\|f\|_{L_{t}^{\rho} L_{x}^{p}\left(\mathbb{R}_{+}^{1+n}\right)} .
$$

For any $t>0$, we have from (3.14) that

$$
\begin{aligned}
\left(W_{j}^{\alpha}\right)^{*} L_{j}^{\alpha} f(t, x) & \\
= & \int_{t}^{\infty} \int_{0}^{\tau} \int_{\mathbb{R}^{n}} e^{i(x \cdot \xi+(\phi(t)-\phi(s))|\xi|)} \overline{b_{j}(\tau, t, \xi)} \partial_{\tau} b_{j}(\tau, s, \xi) \hat{f}(s, \xi) \frac{d \xi}{|\xi|^{2 \alpha}} d s d \tau \\
= & \int_{0}^{t} \int_{\mathbb{R}^{n}} e^{i(x \cdot \xi+(\phi(t)-\phi(s))|\xi|)}\left(\int_{t}^{\infty} \overline{b_{j}(\tau, t, \xi)} \partial_{\tau} b_{j}(\tau, s, \xi) d \tau\right) \hat{f}(s, \xi) \frac{d \xi}{|\xi|^{2 \alpha}} d s \\
& \quad+\int_{t}^{\infty} \int_{\mathbb{R}^{n}} e^{i(x \cdot \xi+(\phi(t)-\phi(s))|\xi|)}\left(\int_{s}^{\infty} \overline{b_{j}(\tau, t, \xi)} \partial_{\tau} b_{j}(\tau, s, \xi) d \tau\right) \hat{f}(s, \xi) \frac{d \xi}{|\xi|^{2 \alpha}} d s .
\end{aligned}
$$

Due to conditions (2.19)-(2.21), one has that the first and second term in (3.22) are essentially $W_{j}^{2 \alpha+\frac{2}{m+2}} f$ and $\left(W_{j}^{2 \alpha+\frac{2}{m+2}}\right)^{*} f$, respectively, where $b \in C^{\infty}\left(\mathbb{R}_{+} \times \mathbb{R}_{+} \times \mathbb{R}^{n}\right)$ satisfies condition (2.19). Then, by applying Theorem 3.1 and estimates (3.15) and (3.16), we have that

$$
\mathrm{I} \lesssim \begin{cases}\lambda_{j}^{(n+1)\left(\frac{1}{p}-\frac{1}{2}\right)-\frac{m}{\mu(m+2)}-\frac{4}{m+2}-2 \operatorname{Re} \alpha}\|f\|_{L_{t}^{r} L_{x}^{p}\left(\mathbb{R}_{+}^{1+n}\right)}^{2} & \text { if } \max \left\{p_{1}, 1\right\}<p \leq 2, \\ \lambda_{j}^{n\left(\frac{2}{p}-1\right)-\frac{6}{m+2}-2 \operatorname{Re} \alpha}\|f\|_{L_{t}^{2} L_{x}^{p}\left(\mathbb{R}_{+}^{1+n}\right)}^{2} & \text { if } p_{1}>1 \text { and } 1<p<p_{1}\end{cases}
$$

which together with (3.21) yields that

$$
\|U(t)\|^{2} \lesssim \begin{cases}\lambda_{j}^{(n+1)\left(\frac{1}{p}-\frac{1}{2}\right)-\frac{m}{\mu(m+2)}-\frac{4}{m+2}-2 \operatorname{Re} \alpha}\|f\|_{L_{t}^{r} L_{x}^{p}\left(\mathbb{R}_{+}^{1+n}\right)}^{2} & \text { if } \max \left\{p_{1}, 1\right\}<p \leq 2, \\ \lambda_{j}^{n\left(\frac{2}{p}-1\right)-\frac{6}{m+2}-2 \operatorname{Re} \alpha}\|f\|_{L_{t}^{2} L_{x}^{p}\left(\mathbb{R}_{+}^{1+n}\right)}^{2} & \text { if } p_{1}>1 \text { and } 1<p<p_{1} .\end{cases}
$$

Note that $\left\|W_{j}^{\alpha} f(t, \cdot)\right\|_{L^{2}\left(\mathbb{R}^{n}\right)}=\|U(t)\|$. Therefore, we have obtained estimates (3.17)-(3.20) which completes the proof of Theorem 3.4. 
Remark 3.5. With similar arguments as in the proof of Theorem 3.4, we have from Theorem 3.1 and estimates (3.15) and (3.16) that the operator $\left(W_{j}^{\alpha}\right)^{*}$ also satisfies the estimates (3.17)-(3.20).

Note that if $r=p$ for $r$ defined in (3.1), then $r=p=p_{0}$. Combining Theorem 3.1 and the kernel estimate (3.10), we obtain boundedness of the operator $W_{j}^{\alpha}$ from $L^{p_{0}}\left(\mathbb{R}_{+}^{1+n}\right)$ to $L^{q}\left(\mathbb{R}_{+}^{1+n}\right)$ for certain $\alpha \in \mathbb{C}$ when $q_{0} \leq q \leq \infty$.

Theorem 3.6. Let $\mu \geq \max \{2, m / 2\}$ and $q_{0} \leq q \leq \infty$. Then

$$
\left\|W_{j}^{\alpha} f\right\|_{L^{q}\left(\mathbb{R}_{+}^{1+n}\right)} \lesssim\|f\|_{L^{p_{0}\left(\mathbb{R}_{+}^{1+n}\right)}}
$$

where

$$
\operatorname{Re} \alpha=n-\frac{2}{m+2}-\left(n+\frac{2}{m+2}\right)\left(\frac{1}{q}+\frac{1}{q_{0}}\right) \text {. }
$$

Proof. (i) Case $q=q_{0}$. Note that

$$
n-\frac{2}{q_{0}}\left(n+\frac{2}{m+2}\right)=\left(\frac{1}{p_{0}}-\frac{1}{2}\right)(n+1)-\frac{m}{\mu(m+2)} .
$$

An application of (3.3) with $r=p$ yields that

$$
\left\|W_{j}^{\alpha} f\right\|_{L^{q_{0}\left(\mathbb{R}_{+}^{1+n}\right)}} \lesssim\|f\|_{L^{p_{0}\left(\mathbb{R}_{+}^{1+n}\right)}}, \quad \operatorname{Re} \alpha=n-\frac{2}{m+2}-\frac{2}{q_{0}}\left(n+\frac{2}{m+2}\right) .
$$

(ii) Case $q=\infty$. In order to derive (3.23), it suffices to show that the integral kernel $K_{j}^{\alpha}$ defined in (3.7) satisfies

$$
\sup _{(t, x) \in \mathbb{R}_{+}^{1+n}} \int_{\mathbb{R}_{+}^{1+n}}\left|K_{j}^{\alpha}(t, \tau, x, y)\right|^{q_{0}} d \tau d y<\infty, \quad \operatorname{Re} \alpha=n-\frac{2}{m+2}-\frac{1}{q_{0}}\left(n+\frac{2}{m+2}\right) .
$$

In fact, from (3.7) we have

$$
W_{j}^{\alpha} f(t, x)=\int_{0}^{t} \int_{\mathbb{R}^{n}} K_{j}^{\alpha}(t, \tau, x, y) f(\tau, y) d y d \tau .
$$

By Hölder's inequality, then

$$
\left\|W_{j}^{\alpha} f\right\|_{L^{\infty}\left(\mathbb{R}_{+}^{1+n}\right)} \lesssim\|f\|_{L^{p_{0}\left(\mathbb{R}_{+}^{1+n}\right)}}, \quad \operatorname{Re} \alpha=n-\frac{2}{m+2}-\frac{1}{q_{0}}\left(n+\frac{2}{m+2}\right) .
$$

Now it remains to derive estimate (3.25). In fact, due to the kernel estimate (3.10), for any $N>n$ and $\alpha \in \mathbb{C}$ with $\operatorname{Re} \alpha=n-\frac{2}{m+2}-\frac{1}{q_{0}}\left(n+\frac{2}{m+2}\right)$, we have by (3.10)

$$
\int_{\mathbb{R}_{+}^{1+n}}\left|K_{j}^{\alpha}(t, \tau, x, y)\right|^{q_{0}} d \tau d y
$$




$$
\begin{aligned}
& \lesssim \lambda_{j}^{\left(\frac{n+1}{2}-\operatorname{Re} \alpha-\frac{m}{\mu(m+2)}-\frac{2}{m+2}\right) q_{0}} \int_{0}^{\infty}\left(\lambda_{j}^{-\frac{2}{m+2}}+|t-\tau|\right)^{-\left(\frac{(m+2)(n-1)}{4}+\frac{m}{2 \mu}\right) q_{0}} d \tau \\
& \quad \times \int_{\mathbb{R}^{n}}\left(1+\lambda_{j}|| x-y|-| \phi(t)-\phi(\tau)||\right)^{-N} d y \\
& \lesssim \lambda_{j}^{\left(\frac{n+1}{2}-\operatorname{Re} \alpha-\frac{m}{\mu(m+2)}-\frac{2}{m+2}\right) q_{0}} \int_{0}^{\infty}\left(\lambda_{j}^{-\frac{2}{m+2}}+|t-\tau|\right)^{-\left(\frac{(m+2)(n-1)}{4}+\frac{m}{2 \mu}\right) q_{0}} d \tau \\
& \quad \times \lambda_{j}^{-1} \int_{0}^{\infty}(1+r)^{-N}\left(\lambda_{j}^{-1} r+|\phi(t)-\phi(\tau)|\right)^{n-1} d r \\
& =\lambda_{j}^{\left(\frac{n+1}{2}-\operatorname{Re} \alpha-\frac{m}{\mu(m+2)}-\frac{2}{m+2}\right) q_{0}-1} \\
& \quad \times \int_{0}^{\infty}\left(\lambda_{j}^{-\frac{2}{m+2}}+|t-\tau|\right)^{-\left(\frac{(m+2)(n-1)}{4}+\frac{m}{2 \mu}\right) q_{0}}\left(\lambda_{j}^{-1}+|\phi(t)-\phi(\tau)|\right)^{n-1} d \tau \\
& \quad \times \int_{0}^{\infty}(1+r)^{-N}\left(\frac{r+\lambda_{j}|\phi(t)-\phi(\tau)|}{1+\lambda_{j}|\phi(t)-\phi(\tau)|}\right)^{n-1} d r \\
& \lesssim \lambda_{j}^{\left(\frac{n+1}{2}-\operatorname{Re} \alpha-\frac{m}{\mu(m+2)}-\frac{2}{m+2}\right) q_{0}-1} \int_{0}^{\infty}\left(\lambda_{j}^{-\frac{2}{m+2}}+|t-\tau|\right)^{-\left(\frac{(m+2)(n-1)}{4}+\frac{m}{2 \mu}\right) q_{0}+\frac{(m+2)(n-1)}{2}} d \tau \\
& \lesssim \lambda_{j}^{\left(n-\operatorname{Re} \alpha-\frac{2}{m+2}\right) q_{0}-n-\frac{2}{m+2}}=1,
\end{aligned}
$$

and hence (3.25) holds.

(iii) Case $q_{0}<q<\infty$. Applying Stein's interpolation theorem, one obtains that estimate (3.23) holds by interpolating between estimates (3.24) and (3.26).

Now we consider boundedness of the operator $W_{j}$ from $L_{t}^{r} L_{x}^{p}\left(S_{T}\right)$ to $L_{t}^{s} L_{x}^{q}\left(S_{T}\right)$, where $1 / p$ is symmetric around $1 / p_{0}$.

Theorem 3.7. Let $n \geq 2$. Further let $p_{1}<p<p_{2}$ if $n=2, m \geq 2$ or if $n \geq 3$, and $1<p<\frac{7 \mu}{4 \mu-2}$ if $n=2, m=1$. Then, for any $\mu \geq \mu_{*}$ and $T>0$,

$$
\left\|W_{j} f\right\|_{L_{t}^{s} L_{x}^{q}\left(S_{T}\right)} \lesssim\|f\|_{L_{t}^{r} L_{x}^{p}\left(S_{T}\right)},
$$

where $r$ is defined as in (3.1) and

$$
\left\{\begin{array}{l}
\frac{1}{q}=\frac{1}{p}-\frac{4}{(m+2)(n+1)}\left(1+\frac{m}{2 \mu}\right), \\
\frac{1}{s}=\frac{(m+2)(n-1)}{4}\left(\frac{1}{2}-\frac{1}{q}\right)+\frac{m}{4 \mu}
\end{array}\right.
$$

Proof. Since $1 / p$ is symmetric around $1 / p_{0}$, by duality it suffices to consider the case $\max \left\{p_{1}, 1\right\}<$ $p \leq p_{0}$.

In order to derive (3.27), we now need a further dyadic decomposition with respect to the time variable $t$. Choose a function $\eta \in C_{c}^{\infty}\left(\mathbb{R}_{+}\right)$such that $0 \leq \eta \leq 1$, supp $\eta \subseteq[1 / 2,2]$, and

$$
\sum_{\ell=-\infty}^{\infty} \eta\left(2^{-\ell} t\right)=1
$$


Let us fix $\lambda=2^{j}$ and set

$$
\eta_{0}(t)=\sum_{k \leq 0} \eta\left(\lambda 2^{-k} t\right), \quad \eta_{\ell}(t)=\eta\left(\lambda 2^{-\ell} t\right) \quad \text { for } \ell \in \mathbb{N} .
$$

Then,

$$
W_{j} f(t, x)=\sum_{k=0}^{\infty} G_{k} f(t, x)
$$

where

$$
G_{k} f(t, x)=\int_{0}^{t} \int_{\mathbb{R}^{n}} e^{i(x \cdot \xi+(\phi(t)-\phi(\tau))|\xi|)} \eta_{k}(t-\tau) b_{j}(t, \tau, \xi) \hat{f}(\tau, \xi) d \xi d \tau .
$$

Hence, to derive (3.27), it suffices to show that, for any $k \in \mathbb{N}_{0}$,

$$
\left\|G_{k} f\right\|_{L_{t}^{s} L_{x}^{q}\left(S_{T}\right)} \lesssim 2^{-\varepsilon_{p} k}\|f\|_{L_{t}^{r} L_{x}^{p}\left(S_{T}\right)}
$$

for some $\varepsilon_{p}>0$. From (3.1) and (3.28), we know that

$$
\frac{(m+2) n}{2}\left(\frac{1}{p}-\frac{1}{q}\right)+\frac{1}{r}-\frac{1}{s}=2 .
$$

Due to scaling invariance, we need to consider only the case $\lambda=1$ (by a change of variable if $\lambda \neq 1$ ). Repeating the arguments which are used to prove (3.2), we get that, for any $k \in \mathbb{N}_{0}$,

$$
\left\|G_{k} f\right\|_{L_{t}^{r^{\prime}} L_{x}^{p^{\prime}\left(S_{T}\right)}} \lesssim 2^{-k\left((m+2)\left(\frac{1}{p}-\frac{1}{2}\right) \frac{n-1}{2}+\frac{m}{2 \mu}\right)}\|f\|_{L_{t}^{r} L_{x}^{p}\left(S_{T}\right)} .
$$

Note that $(m+2)\left(\frac{1}{p}-\frac{1}{2}\right) \frac{n-1}{2}+\frac{m}{2 \mu}>\frac{1}{3}$, since $p \leq p_{0}$.

Furthermore, an immediate consequence of (3.17) for $\alpha=0$ is

$$
\left\|G_{k} f\right\|_{L_{t}^{\infty} L_{x}^{2}\left(S_{T}\right)} \lesssim\|f\|_{L_{t}^{r} L_{x}^{p}\left(S_{T}\right)},
$$

and thus, for any $1<\rho<\infty$,

$$
\left\|G_{k} f\right\|_{L_{t}^{\rho} L_{x}^{2}\left(S_{T}\right)} \lesssim\|f\|_{L_{t}^{r} L_{x}^{p}\left(S_{T}\right)}
$$

Choose

$$
\theta=\frac{4 p(2 \mu+m)}{\mu(m+2)(n+1)(2-p)}-1
$$

Then $0 \leq \theta \leq 1$ and, for the number $q$ from (3.28),

$$
\frac{1}{q}=\frac{\theta}{p^{\prime}}+\frac{1-\theta}{2}
$$

For $s$ from (3.28) and $\theta$ from (3.33), we define $s_{0}$ by

$$
2\left(\frac{1}{s}-\frac{1}{s_{0}}\right)=\theta\left((m+2)\left(\frac{1}{p}-\frac{1}{2}\right) \frac{n-1}{2}+\frac{m}{2 \mu}\right)
$$

and then set $\rho=\rho_{*}$ such that

$$
\frac{1}{s_{0}}=\frac{\theta}{r^{\prime}}+\frac{1-\theta}{\rho_{*}}
$$

Since $2<s<s_{0}$, by interpolating between (3.31) and (3.32) when $\rho=\rho_{*}$, we obtain that

$$
\left\|G_{k} f\right\|_{L_{t}^{s_{0}} L_{x}^{q}\left(S_{T}\right)} \lesssim 2^{-2 k\left(\frac{1}{s}-\frac{1}{s_{0}}\right)}\|f\|_{L_{t}^{r} L_{x}^{p}\left(S_{T}\right)} .
$$


Let $\left\{I_{\ell}\right\}$ be non-overlapping intervals of side length $2^{k}$ and $\bigcup_{\ell} I_{\ell}=\mathbb{R}_{+}$, and denote by $\chi_{I}$ the characteristic function of $I$. In view of (3.29) and the compact support of $\eta_{k}$, we have that if $f(t, x)=$ 0 for $t \notin I_{\ell}$, then $G_{k} f(t, x)=0$ for $t \notin I_{\ell}^{*}$, where $I_{\ell}^{*}$ is the interval with the same center as $I_{\ell}$ but of side length $C_{0} 2^{k}$ with some constant $C_{0}=C_{0}(\eta)>0$. Thus, from Minkowski's inequality

$$
\left\|G_{k} f(t, \cdot)\right\|_{L^{q}\left(\mathbb{R}^{n}\right)}^{s} \leq\left(\sum_{\ell}\left\|G_{k}\left(\chi_{I_{\ell}} f\right)(t, \cdot)\right\|_{L^{q}\left(\mathbb{R}^{n}\right)}\right)^{s} \lesssim \sum_{\ell}\left\|G_{k}\left(\chi_{I_{\ell}} f\right)(t, \cdot)\right\|_{L^{q}\left(\mathbb{R}^{n}\right)}^{s},
$$

Denote $\overline{I_{\ell}^{*}}=I_{\ell}^{*} \cap(0, T)$. Estimate (3.35) together with Hölder's inequality and (3.34) yields that, for any $k \in \mathbb{N}_{0}$,

$$
\begin{aligned}
\left\|G_{k} f\right\|_{L_{t}^{s} L_{x}^{q}\left(S_{T}\right)}^{s} & \lesssim \sum_{\ell}\left\|G_{k}\left(\chi_{I_{\ell}} f\right)\right\|_{L_{t}^{s} L_{x}^{q}\left(\overline{I_{\ell}^{*}} \times \mathbb{R}^{n}\right)}^{s} \\
& \lesssim \sum_{\ell}\left|\overline{I_{\ell}^{*}}\right|^{1-s / s_{0}}\left\|G_{k}\left(\chi_{I_{\ell}} f\right)\right\|_{L_{t}^{s} L_{x}^{q}\left(\overline{I_{\ell}^{*}} \times \mathbb{R}^{n}\right)}^{s} \\
& \lesssim 2^{k\left(1-s / s_{0}\right)} 2^{-2 k s\left(1 / s-1 / s_{0}\right)} \sum_{\ell}\left\|\chi_{I_{\ell}} f\right\|_{L_{t}^{r} L_{x}^{p}\left(S_{T}\right)}^{s} \\
& \lesssim 2^{-k\left(1-s / s_{0}\right)}\|f\|_{L_{t}^{r} L_{x}^{p}\left(S_{T}\right)} .
\end{aligned}
$$

Therefore, we get estimate (3.30) with $\varepsilon_{p}=1-s / s_{0}$ and, hence, (3.27) holds.

By a similar argument as in the proof of Theorem 3.7, we obtain the boundedness of operator $W_{j}$ from $L_{t}^{2} L_{x}^{p}\left(S_{T}\right)$ to $L_{t}^{s} L_{x}^{q}\left(S_{T}\right)$ when $p_{1}>1$ and $1<p<p_{1}$.

Theorem 3.8. Let $n \geq 3$ or $n=2, m \geq 2$. Suppose $1<p<p_{1}$. Then, for $\mu \geq \max \{2, m n / 2\}$ and $T>0$, we have that

where

$$
\left\|W_{j} f\right\|_{L_{t}^{s} L_{x}^{q}\left(S_{T}\right)} \lesssim\|f\|_{L_{t}^{2} L_{x}^{p}\left(S_{T}\right)},
$$

$$
\left\{\begin{array}{l}
\frac{1}{q}=\frac{2 n}{p(n+1)}-\frac{n-1}{2(n+1)}-\frac{m+6 \mu}{\mu(m+2)(n+1)}, \\
\frac{1}{s}=(m+2)\left(\frac{1}{2}-\frac{1}{q}\right)\left(\frac{n-1}{4}\right)+\frac{m}{4 \mu} .
\end{array}\right.
$$

Proof. Note that when $1<p<p_{1}$, we have

$$
(m+2)\left(\frac{1}{p}-\frac{1}{2}\right)\left(\frac{n-1}{2}\right)+\frac{m}{2 \mu}>1 .
$$

Then we can apply similar arguments as in the proof Theorem 3.7 to obtain (3.36). We omit the details.

Remark 3.9. By similar arguments as above one can show that adjoints $\left(W_{j}\right)^{*}$ of $W_{j}$ also satisfy estimates (3.27) and (3.36), respectively, under assumptions (3.28) and (3.37).

\section{MIXED-NORM ESTIMATES FOR THE LINEAR GENERALIZED TRICOMI EQUATION}

In this section, based on the mixed-norm space-time estimates of the Fourier integral operators $W_{j}^{\alpha}$ obtained in Section 3, we shall establish Strichartz-type estimates for the linear generalized Tricomi equation.

First we consider the inhomogeneous equation, i.e., problem (2.3). 
Theorem 4.1. Let $n \geq 2$. Suppose $w$ is a solution of (2.3) in $S_{T}$ for some $T>0$. Then:

(i) For $\mu \geq \mu_{*}$,

$$
\|w\|_{L_{t}^{s} L_{x}^{q}\left(S_{T}\right)} \lesssim\|f\|_{L_{t}^{r} L_{x}^{p}\left(S_{T}\right)}
$$

provided that $p_{1}<p<p_{2}$ if $n \geq 3$ or $n=2, m \geq 2$, and $1<p<7 \mu /(4 \mu-2)$ if $n=2$ and $m=1$. Here $r=r(p, \mu)$ is as in (3.1) and $q$ and s are taken from (3.28).

(ii) For $\mu \geq \max \{2, m / 2\}$,

$$
\|w\|_{L^{q}\left(S_{T}\right)} \lesssim\left\|\left|D_{x}\right|^{\gamma-\gamma_{0}} f\right\|_{L^{p_{0}\left(S_{T}\right)}}, \quad q_{0} \leq q<\infty
$$

where

$$
\left\{\begin{array}{l}
\gamma=\gamma(m, n, q)=\frac{n}{2}-\frac{1}{q}\left(n+\frac{2}{m+2}\right) \\
\gamma_{0}=\gamma_{0}(m, n, \mu)=\frac{1}{q_{0}}\left(n+\frac{2}{m+2}\right)+\frac{2}{m+2}-\frac{n}{2}
\end{array}\right.
$$

(iii) For $\mu \geq \max \{2, m / 2\}, \max \left\{p_{1}, 1\right\}<p \leq 2$, and $0 \leq t \leq T$,

$$
\|w(t, \cdot)\|_{\dot{H}^{\gamma}\left(\mathbb{R}^{n}\right)} \lesssim\|f\|_{L_{t}^{r} L_{x}^{p}\left(S_{T}\right)},
$$

where $r=r(p, \mu)$ is defined in (3.1) and

$$
\gamma=\gamma(m, n, \mu, p)=\frac{2}{m+2}+\frac{m}{2 \mu(m+2)}-\left(\frac{1}{p}-\frac{1}{2}\right) \frac{n+1}{2} .
$$

(iv) For $\mu \geq \max \{2, m / 2\}, \gamma \in \mathbb{R}$, and $0 \leq t \leq T$,

$$
\|w(t, \cdot)\|_{\dot{H}^{\gamma}\left(\mathbb{R}^{n}\right)} \lesssim\left\|\left|D_{x}\right|^{\gamma-\gamma_{0}} f\right\|_{L^{p_{0}\left(S_{T}\right)}},
$$

where $\gamma_{0}$ is from (4.3).

Remark 4.2. If we choose $\mu=\mu_{*}$, then

$$
p_{0}=p_{0}^{*}=\frac{2 \mu_{*}}{\mu_{*}+2}, \quad q_{0}=q_{0}^{*}=\frac{2 \mu_{*}}{\mu_{*}-2},
$$

and for $\gamma$ and $\gamma_{0}$ defined in (4.3),

$$
\gamma\left(m, n, q_{0}^{*}\right)=\gamma_{0}\left(m, n, \mu_{*}\right)=\frac{1}{m+2} .
$$

Thus, we have from (4.2) that

$$
\|w\|_{L^{q_{0}^{*}\left(S_{T}\right)}} \lesssim\|f\|_{L^{p_{0}^{*}\left(S_{T}\right)}},
$$

which, for any $\rho \in \mathbb{R}$, together with $\left[\left|D_{x}\right|^{\rho}, \partial_{t}^{2}-t^{m} \Delta\right]=0$ implies that

$$
\left\|\left|D_{x}\right|^{\rho} w\right\|_{L^{q_{0}^{*}\left(S_{T}\right)}} \lesssim\left\|\left|D_{x}\right|^{\rho} f\right\|_{L^{p_{0}^{*}\left(S_{T}\right)}} \cdot
$$




\section{Proof of Theorem 4.1.}

(i) One obtains (4.1) by applying Proposition 2.1 and Theorem 3.7 directly.

(ii) For $\alpha \in \mathbb{C}$, the Fourier transform of $\left|D_{x}\right|^{\alpha} f(t, x)$ with respect to the variable $x$ is $|\xi|^{\alpha} \hat{f}(t, \xi)$. Thus, we can write $W_{j} f$ as

$$
W_{j} f(t, x)=\int_{0}^{t} \int_{\mathbb{R}^{n}} e^{i(x \cdot \xi+(\phi(t)-\phi(\tau))|\xi|)} \Theta\left(|\xi| / 2^{j}\right) b(t, \tau, \xi)\left(\widehat{\left.D_{x}\right|^{\alpha} f}\right)(\tau, \xi)|\xi|^{-\alpha} d \xi d \tau
$$

and $W_{j}(f)=W_{j}^{\alpha}\left(\left|D_{x}\right|^{\alpha} f\right)$. Therefore, applying Theorem 3.6, we get that

$$
\left\|W_{j} f\right\|_{L^{q}\left(S_{T}\right)}=\left\|W_{j}^{\gamma-\gamma_{0}}\left(\left|D_{x}\right|^{\gamma-\gamma_{0}} f\right)\right\|_{L^{q}\left(S_{T}\right)} \lesssim\left\|\left|D_{x}\right|^{\gamma-\gamma_{0}} f\right\|_{L^{p_{0}\left(S_{T}\right)}},
$$

which together with Proposition 2.1 yields (4.2).

(iii) Note that $\left[\left|D_{x}\right|^{\gamma}, \partial_{t}^{2}-t^{m} \Delta\right]=0$ and then

$$
\left(\partial_{t}^{2}-t^{m} \Delta\right)\left(\left|D_{x}\right|^{\gamma} w\right)=\left|D_{x}\right|^{\gamma} f .
$$

From (ii) we know that $W_{j}\left(\left|D_{x}\right|^{\gamma} f\right)=W_{j}^{-\gamma}(f)$. Thus, for $\gamma=\frac{2}{m+2}+\frac{m}{2 \mu(m+2)}-\left(\frac{1}{p}-\frac{1}{2}\right) \frac{n+1}{2}$, we have from estimate (3.18) that

$$
\left\|W_{j}\left(\left|D_{x}\right|^{\gamma} f\right)(t, \cdot)\right\|_{L^{2}\left(\mathbb{R}^{n}\right)}=\left\|W_{j}^{-\gamma} f(t, \cdot)\right\|_{L^{2}\left(\mathbb{R}^{n}\right)} \lesssim\|f\|_{L_{t}^{r} L_{x}^{p}}
$$

Thus, by (4.6) and Proposition 2.1 it follows that

$$
\left\|\left(\left|D_{x}\right|^{\gamma} w\right)(t, \cdot)\right\|_{L^{2}\left(\mathbb{R}^{n}\right)} \lesssim\|f\|_{L_{t}^{r} L_{x}^{p}}
$$

which together with Plancherel's theorem implies that

$$
\|w(t, \cdot)\|_{\dot{H}^{\gamma}\left(\mathbb{R}^{n}\right)}=\left\||\xi|^{\gamma} \hat{w}(t, \xi)\right\|_{L_{\xi}^{2}\left(\mathbb{R}^{n}\right)}=\left\|\left(\left|D_{x}\right|^{\gamma} w\right)(t, \cdot)\right\|_{L_{x}^{2}\left(\mathbb{R}^{n}\right)} \lesssim\|f\|_{L_{t}^{r} L_{x}^{p}},
$$

and estimate (4.4) holds.

(iv) From (ii) we also know that

$$
W_{j}(g)=W_{j}^{-\gamma_{0}}\left(\left|D_{x}\right|^{-\gamma_{0}} g\right)
$$

In (3.1), we have $r=p=p_{0}$ when $r=p$. Estimate (3.18) for

$$
\alpha=-\gamma_{0}=\left(\frac{1}{p_{0}}-\frac{1}{2}\right) \frac{n+1}{2}-\frac{m}{2 \mu(m+2)}-\frac{2}{m+2}
$$

with $p=p_{0}$ yields that

$$
\left\|W_{j}(g)(t, \cdot)\right\|_{L^{2}\left(\mathbb{R}^{n}\right)}=\left\|W_{j}^{-\gamma_{0}}\left(\left|D_{x}\right|^{-\gamma_{0}} g\right)(t, \cdot)\right\|_{L^{2}\left(\mathbb{R}^{n}\right)} \lesssim\left\|\left|D_{x}\right|^{-\gamma_{0}} g\right\|_{L^{p_{0}\left(S_{T}\right)}},
$$

and then, for $g=\left|D_{x}\right|^{\gamma} f$, where $\gamma \in \mathbb{R}$,

$$
\left\|W_{j}\left(\left|D_{x}\right|^{\gamma} f\right)(t, \cdot)\right\|_{L^{2}\left(\mathbb{R}^{n}\right)} \lesssim\left\|\left|D_{x}\right|^{\gamma-\gamma_{0}} f\right\|_{L^{p_{0}\left(S_{T}\right)}} .
$$

Therefore, one has from Plancherel's theorem, Proposition 2.1, (4.6), and (4.7) that

$$
\|w(t, \cdot)\|_{\dot{H}^{\gamma}\left(\mathbb{R}^{n}\right)}=\left\|\left(\left|D_{x}\right|^{\gamma} w\right)(t, \cdot)\right\|_{L^{2}\left(\mathbb{R}^{n}\right)} \lesssim\left\|\left|D_{x}\right|^{\gamma-\gamma_{0}} f\right\|_{L^{p_{0}\left(S_{T}\right)}}
$$

Hence, estimate (4.5) holds.

In case $n \geq 2$ and $m \geq 2$ if $n=2$, we have a more complete set of inequalities for the solution of the linear generalized Tricomi equation. 
Theorem 4.3. Let $n \geq 3$ or $n=2, m \geq 2$. Suppose $w$ solves (2.3) in $S_{T}$. Then:

(i) For $\mu \geq \max \{2, m n / 2\}$ and $\frac{1}{p_{1}}<\frac{1}{p} \leq \frac{1}{2}+\frac{m+6 \mu}{2 \mu n(m+2)}$,

$$
\|w\|_{L_{t}^{s} L_{x}^{q}\left(S_{T}\right)} \lesssim\|f\|_{L_{t}^{2} L_{x}^{p}\left(S_{T}\right)}
$$

where $q$ and s are defined in (3.37).

(ii) For $\mu \geq \max \{2, m n / 2\}$ and $\frac{1}{2} \leq \frac{1}{p}<\frac{1}{2}+\frac{2 \mu(n-3)+m(3 n-1)}{\mu(m+2)\left(n^{2}-1\right)}$,

$$
\|w\|_{L_{t}^{2} L_{x}^{q}\left(S_{T}\right)} \lesssim\|f\|_{L_{t}^{r} L_{x}^{p}\left(S_{T}\right)}
$$

where $r$ is defined in (3.1) and

$$
\frac{1}{q}=\frac{n+1}{2 n p}+\frac{n-1}{4 n}-\frac{m+6 \mu}{2 \mu(m+2) n} .
$$

(iii) For $\mu \geq \max \left\{2, \frac{m}{2}\right\}$ and $1<p<p_{1}$ and $\gamma=\frac{3}{m+2}-n\left(\frac{1}{p}-\frac{1}{2}\right)$,

$$
\|w(t, \cdot)\|_{\dot{H}^{\gamma}\left(\mathbb{R}^{n}\right)} \lesssim\|f\|_{L_{t}^{2} L_{x}^{p}\left(S_{T}\right)} .
$$

Proof. (i) Note that, under these assumptions,

$$
1<\frac{2 \mu n(m+2)}{\mu n(m+2)+6 \mu+m} \leq p<p_{1}, \quad 2 \leq q<\infty, \quad 2 \leq s<\infty .
$$

Thus, we get estimate (4.8) by applying Proposition 2.1 and Theorem 3.8.

(ii) This will follow from the dual version of Theorem 3.8. Indeed, when

$$
\frac{1}{2} \leq \frac{1}{p}<\frac{1}{2}+\frac{2 \mu(n-3)+m(3 n-1)}{\mu(m+2)\left(n^{2}-1\right)},
$$

then, for $q$ defined in (4.10),

$$
1<\frac{2 \mu(m+2) n}{\mu(m+2) n+6 \mu+m} \leq q^{\prime}<p_{1}
$$

and

$$
\frac{1}{p^{\prime}}=\frac{2 n}{q^{\prime}(n+1)}-\frac{n-1}{2(n+1)}-\frac{m+6 \mu}{\mu(m+2)(n+1)},
$$

For $r$ defined by (3.1), the conjugate exponent $r^{\prime}$ can be expressed by

$$
r^{\prime}=\frac{8 \mu p^{\prime}}{\mu(m+2)(n-1)\left(p^{\prime}-2\right)+2 m p^{\prime}} .
$$

Thus, from Remark 3.9, we have that

$$
\left\|W_{j}^{*} f\right\|_{L_{t}^{r^{\prime}} L_{x}^{p^{\prime}}\left(S_{T}\right)} \lesssim\|f\|_{L_{t}^{2} L_{x}^{q^{\prime}}\left(S_{T}\right)},
$$

and then, by duality,

$$
\left\|W_{j} f\right\|_{L_{t}^{2} L_{x}^{q}\left(S_{T}\right)} \lesssim\|f\|_{L_{t}^{r} L_{x}^{p}\left(S_{T}\right)}
$$

Therefore, from Proposition 2.1 we have that estimate (4.9) holds.

(iii) Note again that $W_{j}\left(\left|D_{x}\right|^{\gamma} f\right)=W_{j}^{-\gamma}(f)$. Then, in view of (4.6) and estimate (3.20) for $\alpha=$ $-\gamma=n\left(\frac{1}{p}-\frac{1}{2}\right)-\frac{3}{m+2}$, one has that estimate (4.11) holds.

Now we consider the homogeneous equation, i.e., problem (2.2). 
Theorem 4.4. Let $n \geq 2$ and $\mu \geq \max \{2, m / 2\}$. Suppose $v$ solves the Cauchy problem (2.2). Then: (i) For $q_{0} \leq q<\infty$,

$$
\|v\|_{L^{q}\left(\mathbb{R}_{+}^{1+n}\right)} \lesssim\|\varphi\|_{\dot{H}^{\gamma}\left(\mathbb{R}^{n}\right)}+\|\psi\|_{\dot{H}^{\gamma-\frac{2}{m+2}\left(\mathbb{R}^{n}\right)}},
$$

where $\gamma=\frac{n}{2}-\frac{(m+2) n+2}{q(m+2)}$.

(ii) For $2 \leq q<\infty$ when $n=2$ and $m=1$, and $2 \leq q<q_{1}$ when $n \geq 2$ and $m \geq 2$ if $n=2$,

$$
\|v\|_{L_{t}^{s} L_{x}^{q}\left(\mathbb{R}_{+}^{1+n}\right)} \lesssim\|\varphi\|_{\dot{H}^{\gamma}\left(\mathbb{R}^{n}\right)}+\|\psi\|_{\dot{H}^{\gamma-\frac{2}{m+2}\left(\mathbb{R}^{n}\right)}},
$$

where

$$
\frac{1}{s}=\frac{(m+2)(n-1)}{4}\left(\frac{1}{2}-\frac{1}{q}\right)+\frac{m}{4 \mu}, \quad \gamma=\frac{n+1}{2}\left(\frac{1}{2}-\frac{1}{q}\right)-\frac{m}{2 \mu(m+2)} .
$$

(iii) For $q_{1}<q<\infty$ as well as $n \geq 2$ and $m \geq 2$ if $n=2$,

$$
\|v\|_{L_{t}^{2} L_{x}^{q}\left(\mathbb{R}_{+}^{1+n}\right)} \lesssim\|\varphi\|_{\dot{H}^{\gamma}\left(\mathbb{R}^{n}\right)}+\|\psi\|_{\dot{H}^{\gamma-\frac{2}{m+2}\left(\mathbb{R}^{n}\right)}},
$$

where $\gamma=n\left(\frac{1}{2}-\frac{1}{q}\right)-\frac{1}{m+2}$.

Proof. The goal is to prove that

$$
\|v\|_{L_{t}^{\sigma} L_{x}^{\rho}\left(\mathbb{R}_{+}^{1+n}\right)} \lesssim\|\varphi\|_{\dot{H}^{\gamma}\left(\mathbb{R}^{n}\right)}+\|\psi\|_{\dot{H}^{\gamma-\frac{2}{m+2}\left(\mathbb{R}^{n}\right)}}
$$

for certain $2 \leq \sigma \leq \infty$ and $2 \leq \rho<\infty$.

Note that

$$
t(1+\phi(t)|\xi|)^{-\frac{m+4}{2(m+2)}} \leq(1+\phi(t)|\xi|)^{-\frac{m}{2(m+2)}}|\xi|^{-\frac{2}{m+2}} \leq(1+\phi(t)|\xi|)^{-\frac{m}{\mu(m+2)}}|\xi|^{-\frac{2}{m+2}} .
$$

In order to establish (4.15), from the expression of the function $v$ in (4.22) together with (2.9) and (2.10) and the estimates of $b_{\ell}(t, \xi)(1 \leq \ell \leq 4)$ in (2.13) and (2.14), it suffices to show that

$$
\|P \varphi\|_{L_{t}^{\sigma} L_{x}^{\rho}\left(\mathbb{R}_{+}^{1+n}\right)} \lesssim\|\varphi\|_{\dot{H}^{\gamma}\left(\mathbb{R}^{n}\right)},
$$

where the operator $P$ is of the form

$$
(P \varphi)(t, x)=\int_{\mathbb{R}^{n}} e^{i(x \cdot \xi+\phi(t)|\xi|)} a(t, \xi) \hat{\varphi}(\xi) d \xi
$$

with $a \in C^{\infty}\left(\mathbb{R}_{+} \times \mathbb{R}^{n}\right)$ and, for any $(t, \xi) \in \mathbb{R}_{+}^{1+n}$,

$$
\left|\partial_{\xi}^{\beta} a(t, \xi)\right| \lesssim(1+\phi(t)|\xi|)^{-\frac{m}{\mu(m+2)}}|\xi|^{-|\beta|} .
$$

Note that $P \varphi$ can be written as

$$
(P \varphi)(t, x)=\int_{\mathbb{R}^{n}} e^{i(x \cdot \xi+\phi(t)|\xi|)} a(t, \xi) \widehat{\left.D_{x}\right|^{\gamma} \varphi}(\xi) \frac{d \xi}{|\xi|^{\gamma}},
$$

and, for $h=\left|D_{x}\right|^{\gamma} \varphi$, by Plancherel's theorem,

$$
\|h\|_{L^{2}\left(\mathbb{R}^{n}\right)}=\left\||\xi|^{\gamma} \hat{\varphi}\right\|_{L^{2}\left(\mathbb{R}^{n}\right)}=\|\varphi\|_{\dot{H}^{\gamma}\left(\mathbb{R}^{n}\right)} .
$$

Therefore, in order to prove (4.16), it suffices to show that the operator $T$, where

$$
(T h)(t, x)=\int_{\mathbb{R}^{n}} e^{i(x \cdot \xi+\phi(t)|\xi|)} a(t, \xi) \hat{h}(\xi) \frac{d \xi}{|\xi|^{\gamma}},
$$


is bounded from $L^{2}\left(\mathbb{R}^{n}\right)$ to $L_{t}^{\sigma} L_{x}^{\rho}\left(\mathbb{R}_{+}^{1+n}\right)$. By duality, it suffices to show that the adjoint $T^{*}$ of $T$,

$$
\left(T^{*} f\right)(x)=\int_{0}^{\infty} \int_{\mathbb{R}^{n}} e^{i(x \cdot \xi-\phi(\tau)|\xi|)} \overline{a(\tau, \xi)}|\xi|^{-\gamma} \hat{f}(\tau, \xi) d \xi d \tau,
$$

satisfies

Note that

$$
\left\|T^{*} f\right\|_{L^{2}\left(\mathbb{R}^{n}\right)} \lesssim\|f\|_{L_{t}^{\sigma^{\prime}} L_{x}^{\rho^{\prime}}\left(\mathbb{R}_{+}^{1+n}\right)} \cdot
$$

$$
\begin{aligned}
\left\|T^{*} f\right\|_{L^{2}\left(\mathbb{R}^{n}\right)}^{2} & =\int_{\mathbb{R}^{n}}\left(T^{*} f\right)(x) \overline{\left(T^{*} f\right)(x)} d x \\
& =\int_{\mathbb{R}_{+}^{1+n}} T T^{*} f(t, x) \overline{f(t, x)} d t d x \leq\left\|T T^{*} f\right\|_{L_{t}^{\sigma} L_{x}^{\rho}}\|f\|_{L_{t}^{\sigma^{\prime} L_{x}^{\rho^{\prime}}}}
\end{aligned}
$$

Thus, in order to get (4.20), we only need to show that

$$
\left\|T T^{*} f\right\|_{L_{t}^{\sigma} L_{x}^{\rho}} \lesssim\|f\|_{L_{t}^{\sigma^{\prime}} L_{x}^{\rho^{\prime}}}
$$

From (4.18) and (4.19), we have that

$$
T T^{*} f(t, x)=\int_{0}^{\infty} \int_{\mathbb{R}^{n}} e^{i(x \cdot \xi+(\phi(t)-\phi(\tau))|\xi|)} a(t, \xi) \overline{a(\tau, \xi)} \hat{f}(\tau, \xi) \frac{d \xi}{|\xi|^{2 \gamma}} d \tau .
$$

By (4.17), we further have that

$$
\left|\partial_{\xi}^{\beta}(a(t, \xi) \overline{a(\tau, \xi)})\right| \lesssim(1+|\phi(t)-\phi(\tau)||\xi|)^{-\frac{m}{\mu(m+2)}}|\xi|^{-|\beta|} .
$$

Thus, by Proposition 2.1, in order to get (4.21), it suffices to show that

$$
\left\|G_{j} f\right\|_{L_{t}^{\sigma} L_{x}^{\rho}} \lesssim\|f\|_{L_{t}^{\sigma^{\prime}} L_{x}^{\rho^{\prime}}}
$$

where the operator $G_{j}$ is defined as

$$
G_{j} f(t, x)=\int_{0}^{\infty} \int_{\mathbb{R}^{n}} e^{i(x \cdot \xi+(\phi(t)-\phi(\tau))|\xi|)} \Theta\left(|\xi| / 2^{j}\right) a(t, \xi) \overline{a(\tau, \xi)} \hat{f}(\tau, \xi) \frac{d \xi}{|\xi|^{2 \gamma}} d \tau .
$$

Note that $G_{j} f$ is essentially $W_{j}^{2 \gamma-\frac{2}{m+2}} f$. Therefore, in order to get (4.14), it suffices to show that

$$
\left\|W_{j}^{2 \gamma-\frac{2}{m+2}} f\right\|_{L_{t}^{\sigma} L_{x}^{\rho}} \lesssim\|f\|_{L_{t}^{\sigma^{\prime}} L_{x}^{\rho^{\prime}}}
$$

We first show (4.12): For $\gamma=\frac{n}{2}-\frac{n(m+2)+2}{q(m+2)}$ and $q=q_{0}$, we have that

$$
\left(2 \gamma-\frac{2}{m+2}\right)=\left(\frac{1}{p_{0}}-\frac{1}{2}\right)(n+1)-\frac{m}{\mu(m+2)}-\frac{2}{m+2} .
$$

Thus, we have from estimate (3.3) when $r=p=p_{0}$ that

$$
\left\|W_{j}^{2 \gamma-\frac{2}{m+2}}\right\|_{L^{q_{0}\left(\mathbb{R}_{+}^{1+n}\right)}} \lesssim\|f\|_{L^{p_{0}\left(\mathbb{R}_{+}^{1+n}\right)}} .
$$

On the other hand, from (2.22) and the compact support of $\Theta$,

$$
\left\|W_{j}^{2 \gamma-\frac{2}{m+2}} f\right\|_{L^{\infty}\left(\mathbb{R}_{+}^{1+n}\right)} \lesssim\|f\|_{L^{1}\left(\mathbb{R}_{+}^{1+n}\right)} .
$$


By interpolation between (4.23) and (4.24), we obtain that

$$
\left\|W_{j}^{2 \gamma-\frac{2}{m+2}} f\right\|_{L^{q}\left(\mathbb{R}_{+}^{1+n}\right)} \lesssim\|f\|_{L^{q^{\prime}\left(\mathbb{R}_{+}^{1+n}\right)}}, \quad q_{0} \leq q \leq \infty .
$$

where $q^{\prime}$ is the conjugate exponent $q$. Therefore, we get estimate (4.12).

Next we derive (4.13): Since

$$
\frac{1}{s}=\frac{(m+2)(n-1)}{4}\left(\frac{1}{2}-\frac{1}{q}\right)+\frac{m}{4 \mu},
$$

we can write

$$
\frac{1}{s^{\prime}}=1-\frac{(m+2)(n-1)}{4}\left(\frac{1}{q^{\prime}}-\frac{1}{2}\right)-\frac{m}{4 \mu}
$$

Thus, when $\gamma=\frac{n+1}{2}\left(\frac{1}{2}-\frac{1}{q}\right)-\frac{m}{2 \mu(m+2)}$, applying estimate (3.3) for $\max \left\{p_{1}, 1\right\}<q^{\prime} \leq 2$, we have

$$
\left\|W_{j}^{2 \gamma-\frac{2}{m+2}} f\right\|_{L_{t}^{s} L_{x}^{q}\left(\mathbb{R}_{+}^{1+n}\right)} \lesssim\|f\|_{L_{t}^{s^{\prime}} L_{x}^{q^{\prime}}\left(\mathbb{R}_{+}^{1+n}\right)}
$$

and, therefore, estimate (4.13) holds.

Finally we prove (4.14): When $\gamma=n\left(\frac{1}{2}-\frac{1}{q}\right)-\frac{1}{m+2}$, we have from (3.5) that, for $p_{1}>1$ and $1<q^{\prime}<p_{1}$,

$$
\left\|W_{j}^{2 \gamma-\frac{2}{m+2}} f\right\|_{L_{t}^{2} L_{x}^{q}\left(\mathbb{R}_{+}^{1+n}\right)} \lesssim\|f\|_{L_{t}^{2} L_{x}^{q^{\prime}}\left(\mathbb{R}_{+}^{1+n}\right)} .
$$

Thus, estimate (4.14) holds.

Combining Theorems 4.1, 4.3, and 4.4, we obtain the following results:

Theorem 4.5. Let $u$ solve the Cauchy problem (2.1) in the strip $S_{T}$. Then

$$
\|u\|_{C_{t}^{0} \dot{H}_{x}^{\gamma}\left(S_{T}\right)}+\|u\|_{L_{t}^{s} L_{x}^{q}\left(S_{T}\right)} \lesssim\|\varphi\|_{\dot{H}^{\gamma}\left(\mathbb{R}^{n}\right)}+\|\psi\|_{\dot{H}^{\gamma-\frac{2}{m+2}\left(\mathbb{R}^{n}\right)}}+\|f\|_{L_{t}^{r} L_{x}^{p}\left(S_{T}\right)}
$$

provided that the exponents $p, q, r$, and s satisfy scaling invariance condition (1.10) and one of the following sets of conditions:

$$
\left\{\begin{array}{l}
\frac{1}{p}-\frac{1}{q}=\frac{4}{(m+2)(n+1)}\left(1+\frac{m}{2 \mu}\right), \\
\frac{1}{s}=\frac{(m+2)(n-1)}{4}\left(\frac{1}{2}-\frac{1}{q}\right)+\frac{m}{4 \mu}, \\
\gamma=\frac{n+1}{2}\left(\frac{1}{2}-\frac{1}{q}\right)-\frac{m}{2 \mu(m+2)},
\end{array}\right.
$$

where $\mu \geq \mu_{*}$

$$
\begin{cases}-\frac{1}{6 \mu}<\gamma<\frac{47}{84}+\frac{25}{42 \mu} & \text { if } n=2, m=1, \\ \left|\gamma-\gamma_{*}\right|<\gamma_{d}=\frac{2(2 \mu-m)(n+1)}{\mu(m+2)(n-1)\left(2 \mu_{*}-m\right)} & \text { if } n \geq 3 \text { or } n=2, m \geq 2,\end{cases}
$$


and

$$
\gamma_{*}=\frac{2}{m+2}+\frac{m}{2 \mu(m+2)}-\frac{(2 \mu-m)(n+1)}{2 \mu\left(2 \mu_{*}-m\right)} .
$$

(ii) $n \geq 3$ or $n=2, m \geq 2$ and $r=2$,

$$
\left\{\begin{array}{l}
\frac{1}{s}=\frac{(m+2)(n-1)}{4}\left(\frac{1}{2}-\frac{1}{q}\right)+\frac{m}{4 \mu} \\
\gamma=\frac{n+1}{2}\left(\frac{1}{2}-\frac{1}{q}\right)-\frac{m}{2 \mu(m+2)}
\end{array}\right.
$$

where $\mu \geq \max \{2, m n / 2\}$ and

$$
-\frac{m}{2 \mu(m+2)} \leq \gamma<\frac{3}{m+2}-\frac{n(2 \mu-m)}{\mu(m+2)(n-1)} .
$$

(iii) $n \geq 3$ or $n=2, m \geq 2$ and $s=2$,

$$
\left\{\begin{array}{l}
\frac{1}{r}=1-\frac{m}{4 \mu}-\frac{(m+2)(n-1)}{4}\left(\frac{1}{p}-\frac{1}{2}\right) \\
\gamma=n\left(\frac{1}{2}-\frac{1}{q}\right)-\frac{1}{m+2}
\end{array}\right.
$$

where $\mu \geq \max \{2, m n / 2\}$ and

$$
\frac{\mu(n+1)-m n}{\mu(m+2)(n-1)}<\gamma<\frac{2}{m+2}+\frac{m}{2 \mu(m+2)} .
$$

Remark 4.6. We can rewrite the conditions of Theorem 4.5 in terms on $q$.

(i) For $\mu \geq \mu_{*}$,

$$
\begin{cases}\frac{8}{63}\left(1-\frac{4}{\mu}\right)<\frac{1}{q} \leq \frac{1}{2} & \text { if } n=2, m=1, \\ \frac{1}{p_{2}}<\frac{1}{q}+\frac{4}{(m+2)(n+1)}\left(1+\frac{m}{2 \mu}\right)<\frac{1}{p_{1}} & \text { if } n \geq 3 \text { or } n=2, m \geq 2 .\end{cases}
$$

(ii) For $\mu \geq \max \{2, m n / 2\}$,

$$
\frac{2 n}{(n+1) p_{1}}-\frac{n-1}{2(n+1)}-\frac{1}{(m+2)(n+1)}\left(6+\frac{m}{\mu}\right)<\frac{1}{q} \leq \frac{1}{2} .
$$

(iii) For $\mu \geq \max \{2, m n / 2\}$,

$$
\frac{1}{2}-\frac{1}{2(m+2) n}\left(6+\frac{m}{\mu}\right)<\frac{1}{q}<\frac{1}{q_{1}} .
$$

Theorem 4.7. Let $u$ solve the Cauchy problem (2.1) in the strip $S_{T}$. Then

$$
\|u\|_{C_{t}^{0} \dot{H}_{x}^{\gamma}\left(S_{T}\right)}+\|u\|_{L^{q}\left(S_{T}\right)} \lesssim\|\varphi\|_{\dot{H}^{\gamma}\left(\mathbb{R}^{n}\right)}+\|\psi\|_{\dot{H}^{\gamma-\frac{2}{m+2}\left(\mathbb{R}^{n}\right)}}+\left\|\left|D_{x}\right|^{\gamma-\gamma_{0}} f\right\|_{L^{p_{0}\left(S_{T}\right)}}
$$


provided that the exponents $p, q, r$, and s satisfy (1.10) and $\mu \geq \max \{2, m / 2\}, q_{0} \leq q<\infty$, where

$$
\gamma=\frac{n}{2}-\frac{n(m+2)+2}{q(m+2)}, \quad \gamma_{0}=\frac{2}{m+2}+\frac{m}{2 \mu(m+2)}-\frac{n+1}{2}\left(\frac{1}{p_{0}}-\frac{1}{2}\right) .
$$

Corollary 4.8. Under the conditions of Theorem 4.7, one has

$$
\begin{aligned}
\|u\|_{C_{t}^{0} \dot{H}_{x}^{\gamma}\left(S_{T}\right)}+\|u\|_{L^{q}\left(S_{T}\right)}+\left\|\left|D_{x}\right|^{\gamma-\frac{1}{m+2}} u\right\|_{L^{q_{0}^{*}\left(S_{T}\right)}} & \\
& \lesssim\|\varphi\|_{\dot{H}^{\gamma}\left(\mathbb{R}^{n}\right)}+\|\psi\|_{\dot{H}^{\gamma-\frac{2}{m+2}\left(\mathbb{R}^{n}\right)}}+\left\|\left|D_{x}\right|^{\gamma-\frac{1}{m+2}} f\right\|_{L^{p_{0}^{*}\left(S_{T}\right)}},
\end{aligned}
$$

where $\gamma=\frac{n}{2}-\frac{(m+2) n+2}{q(m+2)}$ and $q_{0}^{*} \leq q<\infty$.

Proof. This follows by combining estimate (4.29) and Remark 4.2 when $\mu=\mu_{*}$.

An application of Theorem 4.5 yields:

Corollary 4.9. Let $u$ solve the Cauchy problem

$$
\left\{\begin{array}{l}
\partial_{t}^{2} u-t^{m} \Delta u=f g \quad \text { in } S_{T} \\
u(0, \cdot)=\partial_{t} u(0, \cdot)=0
\end{array}\right.
$$

Then, for any $\mu \geq \mu_{*}$ and $0<R \leq \infty$,

$$
\|u\|_{C_{t}^{0} \dot{H}_{x}^{\gamma}\left(S_{T} \cap \Lambda_{R}\right)}+\|u\|_{L_{t}^{s} L_{x}^{q}\left(S_{T} \cap \Lambda_{R}\right)}+\|u\|_{L_{t}^{\infty} L_{x}^{\delta}\left(S_{T} \cap \Lambda_{R}\right)} \lesssim\|f\|_{L_{t}^{\sigma} L_{x}^{\rho}\left(S_{T} \cap \Lambda_{R}\right)}\|g\|_{L_{t}^{s} L_{x}^{q}\left(S_{T} \cap \Lambda_{R}\right)},
$$

where $q$ is as in (4.26),

$$
\begin{aligned}
\rho=\frac{\mu(m+2)(n+1)}{2(2 \mu+m)}, & \sigma=\frac{\mu(n+1)}{2 \mu-m n}, \\
\frac{1}{s}=\frac{(m+2)(n-1)}{4}\left(\frac{1}{2}-\frac{1}{q}\right)+\frac{m}{4 \mu}, & \frac{n}{\delta}=\frac{n}{q}+\frac{2}{m+2}\left(\frac{1}{s}-\frac{m}{4 \mu}\right),
\end{aligned}
$$

and

$$
\Lambda_{R}=\left\{(t, x) \in \mathbb{R}_{+} \times \mathbb{R}^{n}|| x \mid+\phi(t)<R\right\} .
$$

Proof. First we study the case $R=\infty$. Note that (4.33) gives that

$$
n\left(\frac{1}{2}-\frac{1}{\delta}\right)=\frac{n+1}{2}\left(\frac{1}{2}-\frac{1}{q}\right)-\frac{m}{2 \mu(m+2)} .
$$

Applying estimate (4.25) in case (i) together with the Sobolev embedding $\dot{H}^{n\left(\frac{1}{2}-\frac{1}{\delta}\right)}\left(\mathbb{R}^{n}\right) \hookrightarrow L^{\delta}\left(\mathbb{R}^{n}\right)$, we have

$$
\|u\|_{C_{t}^{0} \dot{H}_{x}^{\gamma}\left(S_{T}\right)}+\|u\|_{L_{t}^{s} L_{x}^{q}\left(S_{T}\right)}+\|u\|_{L_{t}^{\infty} L_{x}^{\delta}\left(S_{T}\right)} \lesssim\|f g\|_{L_{t}^{r} L_{x}^{p}\left(S_{T}\right)},
$$

where $\frac{1}{p}=\frac{1}{q}+\frac{1}{\rho}, \frac{1}{r}=\frac{1}{s}+\frac{1}{\sigma}$. In addition, from Hölder's inequality,

$$
\|f g\|_{L_{t}^{r} L_{x}^{p}\left(S_{T}\right)} \leq\|f\|_{L_{t}^{\sigma} L_{x}^{\rho}\left(S_{T}\right)}\|g\|_{L_{t}^{s} L_{x}^{q}\left(S_{T}\right)} .
$$

Thus, estimate (4.31) holds for $R=\infty$.

Now let $R<\infty$. Let $\chi$ denote the characteristic function of $S_{T} \cap \Lambda_{R}$. If $u$ solves $\partial_{t}^{2} u-t^{m} \Delta u=f g$ with vanishing initial data and $u_{\chi}$ solves $\partial_{t}^{2} u_{\chi}-t^{m} \Delta u_{\chi}=\chi f g$ with vanishing initial data, then $u=u_{\chi}$ in $S_{T} \cap \Lambda_{R}$ due to finite propagation speed (see [22]). Therefore, 


$$
\begin{aligned}
\|u\|_{C_{t}^{0} \dot{H}_{x}^{\gamma}\left(S_{T} \cap \Lambda_{R}\right)} & +\|u\|_{L_{t}^{s} L_{x}^{q}\left(S_{T} \cap \Lambda_{R}\right)}+\|u\|_{L_{t}^{\infty} L_{x}^{\delta}\left(S_{T} \cap \Lambda_{R}\right)} \\
& =\left\|u_{\chi}\right\|_{C_{t}^{0} \dot{H}_{x}^{\gamma}\left(S_{T}\right)}+\left\|u_{\chi}\right\|_{L_{t}^{s} L_{x}^{q}\left(S_{T}\right)}+\left\|u_{\chi}\right\|_{L_{t}^{\infty} L_{x}^{\delta}\left(S_{T}\right)} \leq\|\chi f\|_{L_{t}^{\sigma} L_{x}^{\rho}\left(S_{T}\right)}\|\chi g\|_{L_{t}^{s} L_{x}^{q}\left(S_{T}\right)} .
\end{aligned}
$$

Consequently, estimate (4.31) holds.

As another application of Theorem 4.5 we have:

Corollary 4.10. Let $u$ be a solution of

$$
\left\{\begin{array}{l}
\partial_{t}^{2} u-t^{m} \Delta u=F(v) \quad \text { in } S_{T} \\
u(0, \cdot)=\partial_{t} u(0, \cdot)=0 .
\end{array}\right.
$$

If $q<\infty$ and $\frac{1}{m+2} \leq \gamma=\frac{n}{2}-\frac{n(m+2)+2}{q(m+2)} \leq \frac{m+3}{m+2}$, then

$$
\begin{aligned}
\|u\|_{C_{t}^{0} \dot{H}_{x}^{\gamma}\left(S_{T}\right)}+\|u\|_{L^{q}\left(S_{T}\right)}+\left\|\left|D_{x}\right|^{\gamma-\frac{1}{m+2}} u\right\|_{L^{q_{0}^{*}\left(S_{T}\right)}} & \\
& \lesssim\left\|F^{\prime}(v)\right\|_{L^{\frac{\mu_{*}}{2}}\left(S_{T}\right)}\left\|\left|D_{x}\right|^{\gamma-\frac{1}{m+2}} v\right\|_{L^{q_{0}^{*}\left(S_{T}\right)}} .
\end{aligned}
$$

Proof. This follows from estimate (4.30) by taking fractional derivatives. Indeed, for $0 \leq \gamma-$ $\frac{1}{m+2} \leq 1$, one has

$$
\begin{aligned}
\|u\|_{C_{t}^{0} \dot{H}_{x}^{\gamma}\left(S_{T}\right)}+\|u\|_{L^{q}\left(S_{T}\right)} & +\left\|\left|D_{x}\right|^{\gamma-\frac{1}{m+2}} u\right\|_{L^{q_{0}^{*}}\left(S_{T}\right)} \\
& \lesssim\left\|\left|D_{x}\right|^{\gamma-\frac{1}{m+2}}(F(v))\right\|_{L^{p_{0}^{*}\left(S_{T}\right)}} \lesssim\left\|F^{\prime}(v)\right\|_{L^{\frac{\mu_{*}}{2}\left(S_{T}\right)}}\left\|\left|D_{x}\right|^{\gamma-\frac{1}{m+2}} v\right\|_{L^{q_{0}^{*}}\left(S_{T}\right)} .
\end{aligned}
$$

\section{Solvability of THE SEMILINEAR GENERALIZED TRICOMI EQUATION}

In this section, we will apply Theorems 4.5 and 4.7 and Corollaries 4.8 to 4.10 with $\mu=\mu_{*}$ to establish the existence and uniqueness of the solution $u$ of problem (1.1). Thereby, we will use the following iteration scheme: For $j \in \mathbb{N}_{0}$, let $u_{j}$ be the solution of

$$
\left\{\begin{array}{l}
\partial_{t}^{2} u_{j}-t^{m} \Delta u_{j}=F\left(u_{j-1}\right) \quad \text { in } \mathbb{R}_{+} \times \mathbb{R}^{n}, \\
u_{j}(0, \cdot)=\varphi, \partial_{t} u_{j}(0, \cdot)=\psi,
\end{array}\right.
$$

where $u_{-1}=0$. Notice that, for $\mu=\mu_{*}$, the exponents from (4.25) in case (i) are

$$
\gamma_{*}=\frac{1}{m+2}, \quad \gamma_{d}=\frac{2(n+1)}{\mu_{*}(m+2)(n-1)} .
$$

In order to get the existence of solutions of the Cauchy problem (1.1) as stated in Theorems 1.1, 1.4, and 1.5, we need to show that, for the sequences $\left\{u_{j}\right\}_{j=0}^{\infty}$ and $\left\{F\left(u_{j}\right)\right\}_{j=0}^{\infty}$ defined by (5.1), there exist a $T>0$ and a function $u$ such that

$$
\begin{aligned}
& u_{j} \rightarrow u \quad \text { in } L_{\mathrm{loc}}^{1}\left(S_{T}\right) \quad \text { as } j \rightarrow \infty, \\
& F\left(u_{j}\right) \rightarrow F(u) \quad \text { in } L_{\mathrm{loc}}^{1}\left(S_{T}\right) \quad \text { as } j \rightarrow \infty \text {. }
\end{aligned}
$$

From (5.2) and (5.3), one obviously has that the limit function $u$ solves problem (1.1) in $S_{T}$. 
Furthermore, let $u, \tilde{u}$ both solve the Cauchy problem (1.1) in $S_{T}$. Then $v=u-\tilde{u}$ satisfies

$$
\left\{\begin{array}{l}
\partial_{t}^{2} v-t^{m} \Delta v=G(u, \tilde{u}) v \text { in } S_{T}, \\
v(0, \cdot)=\partial_{t} v(0, \cdot)=0
\end{array}\right.
$$

where $G(u, \tilde{u})=\frac{F(u)-F(\tilde{u})}{u-\tilde{u}}$ if $u \neq \tilde{u}$ and $G(u, u)=F^{\prime}(u)$. For certain $s, q \geq 2$, we will show that $v \in L_{t}^{s} L_{x}^{q}\left(S_{T}\right)$ and then

$$
\|v\|_{L_{t}^{s} L_{x}^{q}\left(S_{T}\right)} \leq \frac{1}{2}\|v\|_{L_{t}^{s} L_{x}^{q}\left(S_{T}\right)}
$$

Uniqueness of the solution of the Cauchy problem (1.1) in $S_{T}$ follows.

\subsection{Proof of Theorem 1.1.}

5.1.1. Case $\kappa_{1}<\kappa<\kappa_{*}$. From the assumptions of Theorem 1.1, we have

$$
\gamma=\frac{n+1}{4}-\frac{n+1}{\mu_{*}(\kappa-1)}-\frac{m}{2 \mu_{*}(m+2)}
$$

and

$$
q=\frac{\mu_{*}(\kappa-1)}{2}, \quad \frac{1}{s}=\frac{(m+2)(n-1)}{4}\left(\frac{1}{2}-\frac{1}{q}\right)+\frac{m}{4 \mu_{*}} .
$$

Thus,

$$
\gamma=\frac{n+1}{2}\left(\frac{1}{2}-\frac{1}{q}\right)-\frac{m}{2 \mu_{*}(m+2)}, \quad \frac{1}{m+2}-\frac{2(n+1)}{\mu_{*}(m+2)(n-1)}<\gamma<\frac{1}{m+2} .
$$

Existence. In order to show (5.2), set

$$
H_{j}(T)=\left\|u_{j}\right\|_{C_{t}^{0} \dot{H}_{x}^{\gamma}\left(S_{T}\right)}+\left\|u_{j}\right\|_{L_{t}^{s} L_{x}^{q}\left(S_{T}\right)}, \quad N_{j}(T)=\left\|u_{j}-u_{j-1}\right\|_{L_{t}^{s} L_{x}^{q}\left(S_{T}\right)} .
$$

We claim that there exists a constant $\varepsilon_{0}>0$ small such that

$$
2 T^{\frac{1}{q}-\frac{1}{s}} H_{0}(T) \leq \varepsilon_{0}
$$

and

$$
H_{j}(T) \leq 2 H_{0}(T), \quad N_{j}(T) \leq \frac{1}{2} N_{j-1}(T)
$$

Indeed, from the iteration scheme (5.1), we have

$$
\left(\partial_{t}^{2}-t^{m} \Delta\right)\left(u_{j+1}-u_{k+1}\right)=G\left(u_{j}, u_{k}\right)\left(u_{j}-u_{k}\right) .
$$

Note that in (4.32)

$$
\rho=\sigma=\frac{\mu_{*}}{2}
$$

when $\mu=\mu_{*}$. Thus, from (4.31) and condition (1.2),

$$
\begin{aligned}
\left\|u_{j+1}-u_{k+1}\right\|_{C_{t}^{0} \dot{H}_{x}^{\gamma}\left(S_{T}\right)}+\left\|u_{j+1}-u_{k+1}\right\|_{L_{t}^{s} L_{x}^{q}\left(S_{T}\right)} & \\
& \lesssim\left\|G\left(u_{j}, u_{k}\right)\right\|_{L^{\frac{\mu_{*}^{*}}{2}\left(S_{T}\right)}}\left\|u_{j}-u_{k}\right\|_{L_{t}^{s} L_{x}^{q}\left(S_{T}\right)} \\
& \lesssim\left(\left\|u_{j}\right\|_{L^{q}\left(S_{T}\right)}^{\kappa-1}+\left\|u_{k}\right\|_{L^{q}\left(S_{T}\right)}^{\kappa-1}\right)\left\|u_{j}-u_{k}\right\|_{L_{t}^{s} L_{x}^{q}\left(S_{T}\right)} .
\end{aligned}
$$

Note that $s>q$ for $\kappa<\kappa_{*}$. By Hölder's inequality, we arrive at

$$
\left\|u_{j}\right\|_{L^{q}\left(S_{T}\right)} \leq T^{\frac{1}{q}-\frac{1}{s}}\left\|u_{j}\right\|_{L_{t}^{s} L_{x}^{q}\left(S_{T}\right)} .
$$


Since $u_{-1}=0,(5.11)$ together with (5.12) implies that

$$
\left\|u_{j+1}-u_{0}\right\|_{L_{t}^{s} L_{x}^{q}\left(S_{T}\right)}+\left\|u_{j+1}-u_{0}\right\|_{C_{t}^{0} \dot{H}_{x}^{\gamma}\left(S_{T}\right)} \lesssim T^{(\kappa-1)\left(\frac{1}{q}-\frac{1}{s}\right)}\left\|u_{j}\right\|_{L_{t}^{s} L_{x}^{q}\left(S_{T}\right)}^{\kappa} .
$$

From the Minkowski inequality, we have that there exists an $\varepsilon_{0}$ with $0<\varepsilon_{0} \leq 2^{-2 /(\kappa-1)}$ such that

$$
H_{j+1}(T) \leq H_{0}(T)+\frac{1}{2} H_{j}(T) \quad \text { if } T^{\frac{1}{q}-\frac{1}{s}} H_{j}(T) \leq \varepsilon_{0} .
$$

Therefore, by induction on $j$,

$$
H_{j}(T) \leq 2 H_{0}(T) \quad \text { if } 2 T^{\frac{1}{q}-\frac{1}{s}} H_{0}(T) \leq \varepsilon_{0} .
$$

Taking $k=j-1$ in (5.10), estimates (5.11) to (5.13) yield that

$$
N_{j+1}(T) \leq \frac{1}{2} N_{j}(T) \text { if } 2 H_{0}(T) T^{\frac{1}{q}-\frac{1}{s}} \leq \varepsilon_{0}
$$

which together with (5.13) implies that (5.9) holds as long as (5.8) holds.

Since $u_{-1} \equiv 0$ and $u_{0}$ is a solution of problem (2.2), we have from (4.13) that, for $\varphi \in \dot{H}^{\gamma}\left(\mathbb{R}^{n}\right)$ and $\psi \in \dot{H}^{\gamma-\frac{2}{m+2}}\left(\mathbb{R}^{n}\right)$,

$$
N_{0}(T) \leq H_{0}(T) \lesssim\|\varphi\|_{\dot{H}^{\gamma}\left(\mathbb{R}^{n}\right)}+\|\psi\|_{\dot{H}^{\gamma-\frac{2}{m+2}\left(\mathbb{R}^{n}\right)}}
$$

Thus, by choosing $T>0$ small, (5.8) holds. Consequently, there is a function $u \in C_{t}^{0} \dot{H}_{x}^{\gamma}\left(S_{T}\right) \cap$ $L_{t}^{s} L_{x}^{q}\left(S_{T}\right)$ such that

$$
u_{j} \rightarrow u \quad \text { in } L_{t}^{s} L_{x}^{q}\left(S_{T}\right) \quad \text { as } j \rightarrow \infty,
$$

and, therefore, (5.2) holds. It also follows that $u_{j}$ converges to $u$ almost where. By Fatou's lemma, it follows that

$$
\|u\|_{C_{t}^{0} \dot{H}_{x}^{\gamma}\left(S_{T}\right)}+\|u\|_{L_{t}^{s} L_{x}^{q}\left(S_{T}\right)} \leq \liminf _{j \rightarrow \infty}\left(\left\|u_{j}\right\|_{C_{t}^{0} \dot{H}_{x}^{\gamma}\left(S_{T}\right)}+\left\|u_{j}\right\|_{L_{t}^{s} L_{x}^{q}\left(S_{T}\right)}\right) \leq 2 H_{0}(T),
$$

which shows that estimate (1.4) holds.

Now we prove (5.3). It suffices to show that $F(u)$ is bounded in $L_{t}^{r} L_{x}^{p}\left(S_{T}\right)$ and $F\left(u_{j}\right)$ converges to $F(u)$ in $L_{t}^{r} L_{x}^{p}\left(S_{T}\right)$ as $j \rightarrow \infty$, where $p=q / \kappa$ and $\frac{1}{r}=1-\frac{m}{4 \mu_{*}}-\frac{(m+2)(n-1)}{4}\left(\frac{1}{p}-\frac{1}{2}\right)$. In fact, $r \kappa<s$ if $\kappa<\kappa_{*}$, thus, for $q=p \kappa$, by condition (1.2) and Hölder's inequality, we have

$$
\|F(u)\|_{L_{t}^{r} L_{x}^{p}\left(S_{T}\right)} \lesssim\|u\|_{L_{t}^{r \kappa} L_{x}^{p \kappa}\left(S_{T}\right)}^{\kappa} \lesssim T^{\frac{1}{r}-\frac{\kappa}{s}}\|u\|_{L_{t}^{s} L_{x}^{q}\left(S_{T}\right)}^{\kappa} .
$$

Moreover, in view of $\frac{1}{p}-\frac{1}{q}=\frac{1}{r}-\frac{1}{s}=\frac{2}{\mu_{*}}$, by Hölder's inequality and estimates (5.11)-(5.13) and (5.15), we have

$$
\begin{aligned}
\left\|F\left(u_{j}\right)-F(u)\right\|_{L_{t}^{r} L_{x}^{p}\left(S_{T}\right)} \leq\left\|G\left(u_{j}, u\right)\right\|_{L^{\mu * / 2}\left(S_{T}\right)}\left\|u_{j}-u\right\|_{L_{t}^{s} L_{x}^{q}\left(S_{T}\right)} & \\
& \lesssim T^{(\kappa-1)\left(\frac{1}{q}-\frac{1}{s}\right)} H_{0}(T)^{\kappa-1}\left\|u_{j}-u\right\|_{L_{t}^{s} L_{x}^{q}\left(S_{T}\right)} \lesssim\left\|u_{j}-u\right\|_{L_{t}^{s} L_{x}^{q}\left(S_{T}\right)} .
\end{aligned}
$$

Applying (5.14), we have that $F\left(u_{j}\right)$ converges to $F(u)$ in $L_{t}^{r} L_{x}^{p}\left(S_{T}\right)$ and, therefore, (5.3) holds.

From (5.2) and (5.3), we have that the limit function $\left.u \in C_{t}^{0} \dot{H}_{x}^{\gamma}\left(S_{T}\right)\right) \cap L_{t}^{s} L_{x}^{q}\left(S_{T}\right)$ solves the Cauchy problem (1.1) in $S_{T}$. 
Uniqueness. Suppose $u, \tilde{u} \in C\left([0, T], \dot{H}^{\gamma}\left(\mathbb{R}^{n}\right)\right) \cap L_{t}^{s} L_{x}^{q}\left(S_{T}\right)$ solve the Cauchy problem (1.1) in $S_{T}$. Then $v=u-\tilde{u} \in C\left([0, T], \dot{H}^{\gamma}\left(\mathbb{R}^{n}\right)\right) \cap L_{t}^{s} L_{x}^{q}\left(S_{T}\right)$ is a solution of problem (5.4). From Corollary 4.9, we have that

$$
\begin{aligned}
\|v\|_{L_{t}^{s} L_{x}^{q}\left(S_{T}\right)} & \leq C\left(\|u\|_{L^{q}\left(S_{T}\right)}^{\kappa-1}+\|\tilde{u}\|_{L^{q}\left(S_{T}\right)}^{\kappa-1}\right)\|v\|_{L_{t}^{s} L_{x}^{q}\left(S_{T}\right)} & & \text { (by (4.31) and (1.2)) } \\
& \leq C T^{(\kappa-1)\left(\frac{1}{q}-\frac{1}{s}\right)}\left(\|u\|_{L_{t}^{s} L_{x}^{q}\left(S_{T}\right)}^{\kappa-1}+\|\tilde{u}\|_{L_{t}^{s} L_{x}^{q}\left(S_{T}\right)}^{\kappa-1}\right)\|v\|_{L_{t}^{s} L_{x}^{q}\left(S_{T}\right)} & & \text { (by Hölder's inequality) } \\
& \leq C 2^{\kappa}\left(T^{\frac{1}{q}-\frac{1}{s}} H_{0}(T)\right)^{\kappa-1}\|v\|_{L_{t}^{s} L_{x}^{q}\left(S_{T}\right)} & & \text { (by (5.15)) } \\
& \leq \frac{1}{2}\|v\|_{L_{t}^{s} L_{x}^{q}\left(S_{T}\right)} . & & \text { (by (5.8)) }
\end{aligned}
$$

Thus (5.5) holds and $u=\tilde{u}$ in $S_{T}$.

5.1.2. Case $\kappa_{*} \leq \kappa$ if $n=2$ or $\kappa_{*} \leq \kappa \leq \kappa_{3}$ if $n \geq 3$.

Existence. From the assumptions of Theorem 1.1, we have

$$
\gamma=\frac{n}{2}-\frac{4}{(m+2)(\kappa-1)}, \quad s=q=\frac{\mu_{*}(\kappa-1)}{2} .
$$

Thus,

$$
\frac{1}{m+2} \leq \gamma=\frac{n}{2}-\frac{(m+2) n+2}{q(m+2)} \leq \frac{m+3}{m+2}
$$

To show (5.2), we set

$$
H_{j}(T)=\left\|u_{j}\right\|_{C_{t}^{0} \dot{H}_{x}^{\gamma}\left(S_{T}\right)}+\left\|u_{j}\right\|_{L^{q}\left(S_{T}\right)}+\left\|\left|D_{x}\right|^{\gamma-\frac{1}{m+2}} u_{j}\right\|_{L^{q_{0}^{*}\left(S_{T}\right)}},
$$

and

$$
N_{j}(T)=\left\|u_{j}-u_{j-1}\right\|_{L^{q_{0}^{*}}\left(S_{T} \cap \Lambda_{R}\right)} .
$$

We claim that there exists a constant $\varepsilon_{0}>0$ such that

$$
H_{0}(T) \leq \varepsilon_{0},
$$

and

$$
H_{j}(T) \leq 2 H_{0}(T), \quad N_{j}(T) \leq \frac{1}{2} N_{j-1}(T) .
$$

Indeed, since $u_{-1}=0$, from the iteration scheme (5.1), we have

$$
\left(\partial_{t}^{2}-t^{m} \Delta\right)\left(u_{j+1}-u_{0}\right)=F\left(u_{j}\right) .
$$

Thus, estimate (4.35) together with condition (1.2) yields that, for $0 \leq \gamma-\frac{1}{m+2} \leq 1$,

$$
\begin{aligned}
H_{j+1}(T) & \leq H_{0}(T)+C\left\|F^{\prime}\left(u_{j}\right)\right\|_{L^{\frac{\mu_{*}}{2}}\left(S_{T}\right)}\left\|\left|D_{x}\right|^{\gamma-\frac{1}{m+2}} u_{j}\right\|_{L^{q_{0}^{*}\left(S_{T}\right)}} \\
& \leq H_{0}(T)+C\left\|u_{j}\right\|_{L^{q}\left(S_{T}\right)}^{\kappa-1}\left\|\left|D_{x}\right|^{\gamma-\frac{1}{m+2}} u_{j}\right\|_{L^{q_{0}^{*}\left(S_{T}\right)}} \\
& \leq H_{0}(T)+C H_{j}(T)^{\kappa} .
\end{aligned}
$$

Therefore, by induction, we have that

$$
H_{j}(T) \leq 2 H_{0}(T) \quad \text { if } C 2^{\kappa} H_{0}(T)^{\kappa-1}<1 .
$$

Consequently,

$$
H_{j}(T) \leq 2 H_{0}(T) \quad \text { if } H_{0}(T) \leq \varepsilon_{0}
$$


for some $\varepsilon_{0}>0$ small. Notice that, for $q$ and $s$ from (5.6), when $q=s$, so $q=s=q_{0}^{*}$. Hence, by using estimates (5.11)-(5.13) together with (5.20), we get that for $N_{j}$ defined in (5.16),

$$
N_{j}(T) \leq \frac{1}{2} N_{j-1}(T) \quad \text { if } H_{0}(T) \leq \varepsilon_{0}
$$

Estimates (5.20) and (5.21) tell us that (5.18) holds as long as (5.17) holds. To get (5.17), from estimate (4.30) (with $f=0$ ) we have that, for $\varphi \in \dot{H}^{\gamma}\left(\mathbb{R}^{n}\right)$ and $\psi \in \dot{H}^{\gamma-\frac{2}{m+2}}\left(\mathbb{R}^{n}\right)$,

$$
H_{0}(T) \lesssim\|\varphi\|_{\dot{H}^{\gamma}\left(\mathbb{R}^{n}\right)}+\|\psi\|_{\dot{H}^{\gamma-\frac{2}{m+2}\left(\mathbb{R}^{n}\right)}} \cdot
$$

Due to the continuity of the norm in $L^{q}\left(S_{T}\right)$, (5.17) holds for some $T>0$ small. (If $\|\varphi\|_{\dot{H}^{\gamma}\left(\mathbb{R}^{n}\right)}+$ $\|\psi\|_{\dot{H}^{\gamma-\frac{2}{m+2}\left(\mathbb{R}^{n}\right)}}$ is small, then (5.17) holds for any $T>0$, consequently, we get global existence.)

Note that $q=\mu_{*}(\kappa-1) / 2 \geq q_{0}^{*}$ when $\kappa \geq \kappa_{*}$. Thus, from Hölder's inequality and (5.22),

$$
N_{0}(T)=\left\|u_{0}\right\|_{L^{q_{0}^{*}}\left(S_{T} \cap \Lambda_{R}\right)} \lesssim\left\|u_{0}\right\|_{L^{q}\left(S_{T}\right)} \lesssim H_{0}(T) .
$$

From estimates (5.17), (5.18), and (5.23), we get that there exists a function $u \in C_{t}^{0} \dot{H}_{x}^{\gamma}\left(S_{T}\right) \cap L^{q}\left(S_{T}\right)$ with $\left|D_{x}\right|^{\gamma-\frac{1}{m+2}} u \in L^{q_{0}^{*}}\left(S_{T}\right)$ such that

$$
u_{j} \rightarrow u \quad \text { in } L^{q_{0}^{*}}\left(S_{T} \cap \Lambda_{R}\right) \quad \text { as } j \rightarrow \infty,
$$

and (5.2) holds. Thus, from Fatou's lemma and (5.18), it follows that

$$
\|u\|_{C_{t}^{0} \dot{H}_{x}^{\gamma}\left(S_{T}\right)}+\|u\|_{L^{q}\left(S_{T}\right)}+\left\|\left|D_{x}\right|^{\gamma-\frac{1}{m+2}} u\right\|_{L^{q_{0}^{*}\left(S_{T}\right)}} \leq 2 H_{0}(T)
$$

and $u$ satisfies estimate (1.4).

Since $q=\mu_{*}(\kappa-1) / 2 \geq \kappa$ when $\kappa \geq \kappa_{*}$, we have from condition (1.2) that $F(u)$ is locally integrable for $u \in L^{q}\left(S_{T}\right)$. By Hölder's inequality,

$$
\begin{aligned}
\int_{S_{T} \cap \Lambda_{R}}\left|F\left(u_{j}\right)-F(u)\right| d t d x=\int_{S_{T} \cap \Lambda_{R}}\left|G\left(u_{j}, u\right)\right| \mid & u_{j}-u \mid d t d x \\
& \leq\left\|G\left(u_{j}, u\right)\right\|_{L^{p_{0}^{*}\left(S_{T} \cap \Lambda_{R}\right)}}\left\|u_{j}-u\right\|_{L^{q^{*}}\left(S_{T} \cap \Lambda_{R}\right)} .
\end{aligned}
$$

Note that $p_{0}^{*}<\mu_{*} / 2$. Thus, from condition (1.2) we have that

$$
\begin{aligned}
& \left\|G\left(u_{j}, u\right)\right\|_{L^{p_{0}^{*}\left(S_{T} \cap \Lambda_{R}\right)}} \lesssim\left\|u_{j}\right\|_{L^{p_{0}^{*}(\kappa-1)}\left(S_{T} \cap \Lambda_{R}\right)}^{\kappa-1}+\|u\|_{L^{p_{0}^{*}(\kappa-1)}\left(S_{T} \cap \Lambda_{R}\right)}^{\kappa-1} \\
& \lesssim\left\|u_{j}\right\|_{L^{q}\left(S_{T} \cap \Lambda_{R}\right)}^{\kappa-1}+\|u\|_{L^{q}\left(S_{T} \cap \Lambda_{R}\right)}^{\kappa-1} \lesssim H_{0}(T)^{\kappa-1},
\end{aligned}
$$

which together with (5.24) implies that $F\left(u_{j}\right) \rightarrow F(u)$ in $L_{\text {loc }}^{1}\left(S_{T}\right)$. Hence, (5.3) holds.

From (5.2) and (5.3), we have that the limit function $u \in C_{t}^{0} \dot{H}_{x}^{\gamma}\left(S_{T}\right) \cap L^{q}\left(S_{T}\right)$ with $\left|D_{x}\right|^{\gamma-\frac{1}{m+2}} u \in$ $L^{q_{0}^{*}}\left(S_{T}\right)$ is a weak solution of Cauchy problem (1.1) in $S_{T}$. 
Uniqueness. Suppose $u, \tilde{u} \in C_{t}^{0} \dot{H}_{x}^{\gamma}\left(S_{T}\right) \cap L^{q}\left(S_{T}\right)$ with $\left|D_{x}\right|^{\gamma-\frac{1}{m+2}} u,\left|D_{x}\right|^{\gamma-\frac{1}{m+2}} \tilde{u} \in L^{q_{0}^{*}}\left(S_{T}\right)$ solve the Cauchy problem (1.1) in $S_{T}$. Then $v=u-\tilde{u} \in C_{t}^{0} \dot{H}_{x}^{\gamma}\left(S_{T}\right) \cap L^{q}\left(S_{T}\right)$ is a weak solution of problem (5.4). Thus, it follows from Corollary 4.9 that

$$
\begin{aligned}
\|v\|_{L^{q}\left(S_{T}\right)} & \leq C\left(\|u\|_{L^{q}\left(S_{T}\right)}^{\kappa-1}+\|\tilde{u}\|_{L^{q}\left(S_{T}\right)}^{\kappa-1}\right)\|v\|_{L^{q}\left(S_{T}\right)} & & (\text { by }(4.31) \text { and (1.2)) } \\
& \leq C 2^{\kappa} H_{0}(T)^{\kappa-1}\|v\|_{L^{q}\left(S_{T}\right)} & & (\text { by (5.25)) } \\
& \leq \frac{1}{2}\|v\|_{L_{t}^{s} L_{x}^{q}\left(S_{T}\right)} & & (\text { by }(5.17))
\end{aligned}
$$

Thus (5.5) holds and $u=\tilde{u}$ in $S_{T}$.

5.1.3. Case $n \geq 3$ and $\kappa>\kappa_{3}, \kappa \in \mathbb{N}$.

Existence. From the assumptions of Theorem 1.1, we have

$$
\gamma=\frac{n}{2}-\frac{4}{(m+2)(\kappa-1)}, \quad s=q=\frac{\mu_{*}(\kappa-1)}{2}, \quad F(u)= \pm u^{\kappa}
$$

and

$$
\gamma=\frac{n}{2}-\frac{(m+2) n+2}{q(m+2)}>1+\frac{1}{m+2}
$$

To verify (5.2), we set

$$
H_{j}(T)=\left\|u_{j}\right\|_{C_{t}^{0} \dot{H}_{x}^{\gamma}\left(S_{T}\right)}+\sup _{q_{0}^{*} \leq \tau \leq \frac{\mu_{*}(\kappa-1)}{2}}\left\|\left|D_{x}\right|^{\frac{(m+2) n+2}{\tau(m+2)}-\frac{4}{(m+2)(\kappa-1)}} u_{j}\right\|_{L^{\tau}\left(S_{T}\right)}
$$

and

$$
N_{j}(T)=\left\|u_{j}-u_{j-1}\right\|_{L^{q_{0}^{*}}\left(S_{T} \cap \Lambda_{R}\right)} .
$$

We claim that there exists a constant $\varepsilon_{0}>0$ such that

$$
H_{0}(T) \leq \varepsilon_{0}
$$

and

$$
H_{j}(T) \leq 2 H_{0}(T), \quad N_{j}(T) \leq \frac{1}{2} N_{j-1}(T) .
$$

In fact, applying Minkowski's inequality and estimate (4.30) (with $\varphi=\psi=0$ ),

$$
H_{j+1}(T) \leq H_{0}(T)+C \sup _{q_{0}^{*} \leq \tau \leq \mu_{*}(\kappa-1) / 2}\left\|\left|D_{x}\right|^{\frac{n}{2}-\frac{1}{m+2}-\frac{4}{(m+2)(\kappa-1)}}\left(u_{j}^{\kappa}\right)\right\|_{L^{p_{0}^{*}\left(S_{T}\right)}} .
$$

Note that $\alpha=\frac{n}{2}-\frac{1}{m+2}-\frac{4}{(m+2)(\kappa-1)}>1$ when $\kappa>\kappa_{3}$. Thus, $\left|D_{x}\right|^{\alpha}\left(u_{j}^{\kappa}\right)$ can be expressed as a finite linear combination of $\prod_{\ell=1}^{\kappa}\left|D_{x}\right|^{\alpha_{\ell}} u_{j}$, where $0 \leq \alpha_{\ell} \leq \alpha(1 \leq \ell \leq \kappa)$ and $\sum_{\ell=1}^{\kappa} \alpha_{\ell}=\alpha$. By Hölder's inequality, $\left\|\left|D_{x}\right|^{\alpha}\left(u_{j}^{\kappa}\right)\right\|_{L^{p_{0}^{*}\left(S_{T}\right)}}$ is dominated by a finite sum of terms of the form $\prod_{\ell=1}^{\kappa}\left\|\left|D_{x}\right|^{\alpha_{\ell}} u_{j}\right\|_{L^{\tau_{\ell}\left(S_{T}\right)}}$, where $\sum_{\ell=1}^{\kappa} 1 / \tau_{\ell}=1 / p_{0}^{*}$. We choose $\tau_{\ell}$ so that

$$
\alpha_{\ell}=\frac{n(m+2)+2}{\tau_{\ell}(m+2)}-\frac{4}{(m+2)(\kappa-1)}
$$


Then

$$
q_{0}^{*} \leq \tau_{\ell} \leq \frac{\mu_{*}(\kappa-1)}{2}, \quad \sum_{\ell=1}^{\kappa} \frac{1}{\tau_{\ell}}=\frac{1}{p_{0}^{*}}
$$

and, therefore,

$$
\left\|\left|D_{x}\right|^{\alpha_{\ell}} u_{j}\right\|_{L^{\tau} \ell\left(S_{T}\right)} \leq H_{j}(T),
$$

which together with (5.28) yields that

$$
H_{j+1}(T) \leq H_{0}(T)+C_{\kappa} H_{j}(T)^{\kappa} .
$$

By induction, we have that

$$
H_{j}(T) \leq 2 H_{0}(T) \quad \text { if } H_{0}(T) \leq \varepsilon_{0} .
$$

For $q$ and $s$ from (5.6), when $q=s$, so $q=s=q_{0}^{*}$. Hence, by estimates (5.11)-(5.13) and together with (5.29), we get that

$$
N_{j}(T) \leq \frac{1}{2} N_{j-1}(T) \quad \text { if } H_{0}(T) \leq \varepsilon_{0} .
$$

From (5.29) and (5.30), we get that (5.27) holds as long as (5.26) holds.

Note that

$$
\frac{n(m+2)+2}{\tau(m+2)}-\frac{4}{(m+2)(\kappa-1)}=0,
$$

for $\tau=\mu_{*}(\kappa-1) / 2$ and

$$
\frac{n(m+2)+2}{\tau(m+2)}-\frac{4}{(m+2)(\kappa-1)}=\gamma-\frac{1}{m+2} .
$$

for $\tau=q_{0}^{*}$. On the other hand, we have from (4.30) (with $f=0$ ) that, for $\varphi \in \dot{H}^{\gamma}\left(\mathbb{R}^{n}\right.$ ) and $\psi \in \dot{H}^{\gamma-\frac{2}{m+2}}\left(\mathbb{R}^{n}\right)$,

$$
\begin{aligned}
&\left\|u_{0}\right\|_{C_{t}^{0} \dot{H}_{x}^{\gamma}\left(S_{T}\right)}+\left\|u_{0}\right\|_{L}{ }_{L}^{\frac{\mu_{*}(\kappa-1)}{2}\left(S_{T}\right)} \\
& \lesssim\|\varphi\|_{\dot{H}^{\gamma}\left(\mathbb{R}^{n}\right)}+\|\psi\|_{\dot{H}^{\gamma-\frac{2}{m+2}\left(\mathbb{R}^{n}\right)}} .
\end{aligned}
$$

By interpolation together with (5.31)-(5.33), we conclude that

$$
H_{0}(T) \lesssim\|\varphi\|_{\dot{H}^{\gamma}\left(\mathbb{R}^{n}\right)}+\|\psi\|_{\dot{H}^{\gamma-\frac{2}{m+2}\left(\mathbb{R}^{n}\right)}} .
$$

It follows that (5.26) holds by choosing $T>0$ small. (We can take $T=\infty$ if $\|\varphi\|_{\dot{H}^{\gamma}\left(\mathbb{R}^{n}\right)}+$ $\|\psi\|_{\dot{H}^{\gamma-\frac{2}{m+2}\left(\mathbb{R}^{n}\right)}}$ is small which then yields global existence.)

From Hölder's inequality and (5.31),

$$
N_{0}(T)=\left\|u_{0}\right\|_{L^{q_{0}^{*}}\left(S_{T} \cap \Lambda_{R}\right)} \leq C_{R}\left\|u_{0}\right\|_{L^{\frac{\mu_{*}(\kappa-1)}{2}\left(S_{T}\right)}} \leq C_{R} H_{0}(T)<\infty .
$$

Therefore, we have from (5.27), (5.26), and (5.34) that there exists a function $u \in C_{t}^{0} \dot{H}_{x}^{\gamma}\left(S_{T}\right) \cap L^{q}\left(S_{T}\right)$ with $\left|D_{x}\right|^{\gamma-\frac{1}{m+2}} u \in L^{q_{0}^{*}}\left(S_{T}\right)$ such that

$$
u_{j} \rightarrow u \quad \text { in } L^{q_{0}^{*}}\left(S_{T} \cap \Lambda_{R}\right) \quad \text { as } j \rightarrow \infty,
$$

and, therefore, (5.2) holds. Thus, from Fatou's lemma and (5.27),

$$
\|u\|_{C_{t}^{0} \dot{H}_{x}^{\gamma}\left(S_{T}\right)}+\|u\|_{L^{q}\left(S_{T}\right)}+\left\|\left|D_{x}\right|^{\gamma-\frac{1}{m+2}} u\right\|_{L^{q_{0}^{*}\left(S_{T}\right)}} \leq 2 H_{0}(T)
$$


and $u$ satisfies estimate (1.4).

Note that $q=\mu_{*}(\kappa-1) / 2 \geq \kappa$ when $\kappa>\kappa_{3}$. Thus, for $u \in L^{q}\left(S_{T}\right)$, by Hölder's inequality and condition (1.2), we get that $F(u)$ is locally integrable and $F\left(u_{j}\right)$ convergences to $F(u)$ in $L_{\text {loc }}^{1}\left(S_{T}\right)$, and hence (5.3) holds.

Applying (5.2), (5.3), it follows that the limit function $u \in C_{t}^{0} \dot{H}_{x}^{\gamma}\left(S_{T}\right) \cap L^{q}\left(S_{T}\right)$ with $\left|D_{x}\right|^{\gamma-\frac{1}{m+2}} u \in$ $L^{q_{0}^{*}}\left(S_{T}\right)$ is a weak solution of the Cauchy problem (1.1) in $S_{T}$.

Uniqueness. This follows from the same arguments as in 5.1.2.

5.2. Proof of Theorem 1.4. From the assumption of Theorem 1.4, we have

$$
\begin{aligned}
& \gamma=\frac{n}{2}-\frac{4}{(m+2)(\kappa-1)} \\
& \frac{1}{q}=\frac{1}{(m+2)(n+1)}\left(\frac{8}{\kappa-1}-\frac{m}{\mu_{*}}\right)-\frac{n-1}{2(n+1)},
\end{aligned}
$$

and

$$
\frac{1}{s}=\frac{(m+2)(n-1)}{4}\left(\frac{1}{2}-\frac{1}{q}\right)+\frac{m}{4 \mu_{*}}
$$

Thus,

$$
\gamma=\left(\frac{n+1}{2}\right)\left(\frac{1}{2}-\frac{1}{q}\right)-\frac{m}{2 \mu_{*}(m+2)}
$$

and

$$
\frac{1}{m+2} \leq \gamma<\frac{1}{m+2}+\frac{2(n+1)}{\mu_{*}(m+2)(n-1)},
$$

where $\kappa_{*} \leq \kappa<\kappa_{2}$.

To show (5.2), we set

$$
H_{j}(T)=\left\|u_{j}\right\|_{C_{t}^{0} \dot{H}_{x}^{\gamma}\left(S_{T}\right)}+\left\|u_{j}\right\|_{L_{t}^{s} L_{x}^{q}\left(S_{T}\right)}+\left\|u_{j}-u_{0}\right\|_{L_{t}^{\infty} L_{x}^{\delta}\left(S_{T}\right)}
$$

and

where

$$
N_{j}(T)=\left\|u_{j}-u_{j-1}\right\|_{L_{t}^{s} L_{x}^{q}\left(S_{T}\right)},
$$

$$
\frac{1}{s}+\frac{(m+2) n}{2 q}=\frac{(m+2) n}{2 \delta}=\frac{m+2}{2}\left(\frac{n}{2}-\gamma\right) .
$$

We claim that there exist a constant $\varepsilon_{0}>0$ and a $\theta \in[0,1]$ such that

$$
2 H_{0}(T)^{\theta}\left(2 H_{0}(T)+\left\|u_{0}\right\|_{L_{t}^{\infty} L_{x}^{\delta}\left(S_{T}\right)}\right)^{1-\theta} \leq \varepsilon_{0}
$$

and

$$
H_{j}(T) \leq 2 H_{0}(T), \quad N_{j}(T) \leq \frac{1}{2} N_{j-1}(T) .
$$

Indeed, due to (5.36), from Sobolev's embedding theorem we have that

$$
\|u(t, \cdot)\|_{L^{\delta}\left(\mathbb{R}^{n}\right)} \lesssim\|u(t, \cdot)\|_{\dot{H}^{\gamma}\left(\mathbb{R}^{n}\right)} .
$$

Applying Hölder's inequality, we get that

$$
\left\|u_{j}\right\|_{L} \frac{\mu_{*(\kappa-1)}^{2}\left(S_{T}\right)}{2} \leq\left\|u_{j}\right\|_{L_{t}^{s} L_{x}^{q}\left(S_{T}\right)}^{\theta}\left\|u_{j}\right\|_{L_{t}^{\infty} L_{x}^{\delta}\left(S_{T}\right)}^{1-\theta},
$$


where $\theta=\frac{2}{n(m+2)+2}+\frac{4 n(m+2)}{\mu_{*}(m+2)(n-1)(q-2)+2 m q}$. Note that $0 \leq \theta \leq 1$ for $\gamma \geq \frac{1}{m+2}$.

By the same arguments as in the proof of Theorem 1.1, we get that (5.37) and (5.38) hold. Consequently, (5.2) and (5.3) also hold. Hence, the limit $u \in C_{t}^{0} \dot{H}_{x}^{\gamma}\left(S_{T}\right) \cap L_{t}^{s} L_{x}^{q}\left(S_{T}\right)$ of the sequence $\left\{u_{j}\right\}$ is a solution of the Cauchy problem (1.1) in $S_{T}$. Moreover, by Fatou's lemma and (5.38), we have that

$$
\|u\|_{C_{t}^{0} \dot{H}_{x}^{\gamma}\left(S_{T}\right)}+\|u\|_{L_{t}^{s} L_{x}^{q}\left(S_{T}\right)} \leq 2 H_{0}(T),
$$

which together with (5.37) yields that $u$ satisfies estimate (1.4).

Further, by the same arguments as in the proof of Theorem 1.1, it follows that if both $u, \tilde{u}$ solve the Cauchy problem (1.1) in $S_{T}$, then $u=\tilde{u}$ in $S_{T}$.

5.3. Proof of Theorem 1.5. From the assumptions of Theorem 1.5, we have

$$
\gamma=\frac{n+1}{2}\left(\frac{1}{2}-\frac{1}{q}\right)-\frac{m}{2 \mu_{*}(m+2)}
$$

and

$$
-\frac{m}{2 \mu_{*}(m+2)} \leq \gamma<\frac{1}{m+2}-\frac{2(n+1)}{\mu_{*}(m+2)(n-1)}=\frac{3}{m+2}-\frac{n\left(2 \mu_{*}-m\right)}{\mu_{*}(m+2)(n-1)} .
$$

To verify (5.2), we set

$$
H_{j}(T)=\left\|u_{j}\right\|_{C_{t}^{0} \dot{H}_{x}^{\gamma}\left(S_{T}\right)}+\left\|u_{j}\right\|_{L_{t}^{s} L_{x}^{q}\left(S_{T}\right)}, \quad N_{j}(T)=\left\|u_{j}-u_{j-1}\right\|_{L_{t}^{s} L_{x}^{q}\left(S_{T}\right)} .
$$

Let $p=q / \kappa$. Then

$$
\frac{2 n}{(n+1) p}=\frac{1}{q}+\frac{6 \mu+m}{\mu(m+2)(n+1)}-\frac{n-1}{2(n+1)} .
$$

Thus we can apply Theorem 4.5 in case (ii) together with Hölder's inequality to find that

$$
\begin{aligned}
\left\|u_{j+1}-u_{k+1}\right\|_{C_{t}^{0} \dot{H}_{x}^{\gamma}\left(S_{T}\right)} & +\left\|u_{j+1}-u_{k+1}\right\|_{L_{t}^{s} L_{x}^{q}\left(S_{T}\right)} \\
& \lesssim\left\|F\left(u_{j}\right)-F\left(u_{k}\right)\right\|_{L_{t}^{2} L_{x}^{p}\left(S_{T}\right)} \lesssim\left\|G\left(u_{j}, u_{k}\right)\right\|_{L_{t}^{\rho} L_{x}^{\sigma}\left(S_{T}\right)}\left\|u_{j}-u_{k}\right\|_{L_{t}^{s} L_{x}^{q}\left(S_{T}\right)},
\end{aligned}
$$

where $1 / \rho=1 / 2-1 / s, 1 / \sigma=1 / p-1 / q=(\kappa-1) / q$.

Note that $s>(\kappa-1) \rho$ when $\gamma<\frac{1}{m+2}-\frac{2(n+1)}{\mu_{*}(m+2)(n-1)}$. Due to condition (1.2) and Hölder's inequality,

$$
\begin{aligned}
\left\|G\left(u_{j}, u_{k}\right)\right\|_{L_{t}^{\rho} L_{x}^{\sigma}\left(S_{T}\right)} & \lesssim\left\|u_{j}\right\|_{L_{t}^{\rho(\kappa-1)} L_{x}^{q}\left(S_{T}\right)}^{\kappa-1}+\left\|u_{k}\right\|_{L_{t}^{\rho(\kappa-1)} L_{x}^{q}\left(S_{T}\right)}^{\kappa-1} \\
& \lesssim T^{\frac{1}{2}-\frac{1}{s}}\left(\left\|u_{j}\right\|_{L_{t}^{s} L_{x}^{q}\left(S_{T}\right)}^{\kappa-1}+\left\|u_{k}\right\|_{L_{t}^{s} L_{x}^{q}\left(S_{T}\right)}^{\kappa-1}\right)
\end{aligned}
$$

As in the proof of Theorem 1.1, we get that

$$
H_{j}(T) \leq 2 H_{0}(T), \quad N_{j}(T) \leq \frac{1}{2} N_{j-1}(T)
$$

and

$$
N_{0}(T) \leq H_{0}(T) T^{1 / 2-\kappa / s} \leq \varepsilon_{0},
$$

for $\varepsilon_{0}>0$ small by choosing $T>0$ small. Therefore, there is a function $u \in C_{t}^{0} \dot{H}_{x}^{\gamma}\left(S_{T}\right) \cap L_{t}^{s} L_{x}^{q}\left(S_{T}\right)$ such that

$$
u_{j} \rightarrow u \quad \text { in } L_{t}^{s} L_{x}^{q}\left(S_{T}\right) \text { as } j \rightarrow \infty
$$


and (5.2) holds. Combining Fatou's lemma and (5.39), we see that

$$
\|u\|_{C_{t}^{0} \dot{H}_{x}^{\gamma}\left(S_{T}\right)}+\|u\|_{L_{t}^{s} L_{x}^{q}\left(S_{T}\right)} \leq 2 H_{0}(T) .
$$

Together with (5.40) we get that $u$ satisfies estimate (1.4).

Moreover, since $2 \kappa>s$, by condition (1.2) and Hölder's inequality, we have that, for $p=q / \kappa$,

$$
\|F(u)\|_{L_{t}^{2} L_{x}^{p}\left(S_{T}\right)} \lesssim\|u\|_{L_{t}^{2 \kappa} L_{x}^{q}\left(S_{T}\right)}^{\kappa} \lesssim T^{\frac{1}{2}-\frac{\kappa}{s}}\|u\|_{L_{t}^{s} L_{x}^{q}\left(S_{T}\right)}^{\kappa}
$$

and

$$
\begin{aligned}
\left\|F\left(u_{j}\right)-F(u)\right\|_{L_{t}^{2} L_{x}^{p}\left(S_{T}\right)} & \lesssim T^{\frac{1}{2}-\frac{1}{s}}\left(\left\|u_{j}\right\|_{L_{t}^{s} L_{x}^{q}\left(S_{T}\right)}^{\kappa-1}+\|u\|_{L_{t}^{s} L_{x}^{q}\left(S_{T}\right)}^{\kappa-1}\right)\left\|u_{j}-u\right\|_{L_{t}^{s} L_{x}^{q}\left(S_{T}\right)} \\
& \lesssim T^{\frac{1}{2}-\frac{1}{s}} H_{0}(T)^{\kappa-1}\left\|u_{j}-u\right\|_{L_{t}^{s} L_{x}^{q}\left(S_{T}\right)},
\end{aligned}
$$

Therefore, $F(u) \in L_{t}^{2} L_{x}^{q / \kappa}\left(S_{T}\right)$ and $F\left(u_{j}\right) \rightarrow F(u)$ in $L_{t}^{2} L_{x}^{q / \kappa}\left(S_{T}\right)$ as $j \rightarrow \infty$, hence (5.3) holds. Consequently, the limit function $u \in C_{t}^{0} \dot{H}_{x}^{\gamma}\left(S_{T}\right) \cap L_{t}^{s} L_{x}^{q}\left(S_{T}\right)$ solves the Cauchy problem (1.1) in $S_{T}$.

Now suppose $u, \tilde{u} \in C_{t}^{0} \dot{H}_{x}^{\gamma}\left(S_{T}\right) \cap L_{t}^{s} L_{x}^{q}\left(S_{T}\right)$ both solve the Cauchy problem (1.1) in $S_{T}$. Then $v=u-\tilde{u} \in C_{t}^{0} \dot{H}_{x}^{\gamma}\left(S_{T}\right) \cap L_{t}^{s} L_{x}^{q}\left(S_{T}\right)$ is a solution of Eq. (5.4). Applying Theorem 4.5 in case (ii) and Hölder's inequality, it follows that

$$
\begin{aligned}
\|v\|_{L_{t}^{s} L_{x}^{q}\left(S_{T}\right)} & \leq C\|G(u, \tilde{u}) v\|_{L_{t}^{2} L_{x}^{p}\left(S_{T}\right)} \leq C T^{\frac{1}{2}-\frac{1}{s}}\left(\|u\|_{L_{t}^{s} L_{x}^{q}\left(S_{T}\right)}^{\kappa-1}+\|\tilde{u}\|_{L_{t}^{s} L_{x}^{q}\left(S_{T}\right)}^{\kappa-1}\right)\|v\|_{L_{t}^{s} L_{x}^{q}\left(S_{T}\right)} \\
& \leq C T^{\frac{1}{2}-\frac{1}{s}} H_{0}(T)^{\kappa-1}\|v\|_{L_{t}^{s} L_{x}^{q}\left(S_{T}\right)} \leq \frac{1}{2}\|v\|_{L_{t}^{s} L_{x}^{q}\left(S_{T}\right)} .
\end{aligned}
$$

Thus (5.5) holds and $u=\tilde{u}$ in $S_{T}$.

\section{REFERENCES}

[1] J. Barros-Neto and I.-M. Gelfand, Fundamental solutions for the Tricomi operator. I, II, Duke Math. J. 98 (1999), 465-483; 111 (2002), 561-584.

[2] M. Beals, Singularities due to cusp interactions in nonlinear waves. Nonlinear hyperbolic equations and field theory (Lake Como, 1990), pp. 36-51, Pitman Res. Notes Math. Ser., 253, Longman, Harlow, 1992.

[3] L. Bers, Mathematical aspects of subsonic and transonic gas dynamics, Surveys Appl. Math., 3, Wiley, New York, 1958.

[4] M. Dreher and I. Witt, Sharp energy estimates for a class of weakly hyperbolic operators. New trends in the theory of hyperbolic equations, pp. 449-511, Oper. Theory Adv. Appl., 159, Birkhäuser, Basel, 2005.

[5] A. Erdelyi, W. Magnus, F. Oberhettinger, and F.-G. Tricomi, Higher transcendental functions. Vol.1. McGraw, New York, 1953.

[6] P. Germain, Remarks on the theory of partial differential equations of mixed type and applications to the study of transonic flow, Comm. Pure Appl. Math. 7 (1954), 117-143.

[7] D.-Y. He, I. Witt, and H.-Y. Yin, On the global solution problem for semilinear generalized Tricomi equations, I, to appear in Calc. Var. Partial Differential Equations.

[8] L. Kapitanski, Weak and yet weaker solutions of semilinear wave equations, Comm. Partial Differential Equations 19 (1994), 1629-1676.

[9] H. Lindbald, Counterexamples to local existence for quasilinear wave equations, Math. Res. Lett. 5 (1998), 605-622.

[10] H. Lindblad and C.-D. Sogge, On existence and scattering with minimal regularity for semilinear wave equations, J. Funct. Anal. 130 (1995), 357-426.

[11] D. Lupo and K.-R. Payne, Conservation laws for equations of mixed elliptic-hyperbolic and degenerate types, Duke Math. J. 127 (2005), 251-290.

[12] _ Critical exponents for semilinear equations of mixed elliptic-hyperbolic and degenerate types, Comm. Pure Appl. Math. 56 (2003), 403-424.

[13] C.-S. Morawetz, Mixed equations and transonic flow, J. Hyperbolic Differ. Equ. 1 (2004), 1-26. 
[14] G. Ponce and T. C. Sideris, Local regularity of nonlinear wave equations in three space dimensions, Comm. Partial Differential Equations 18 (1993), 169-177.

[15] Z.-P. Ruan, I. Witt, and H.-C. Yin, The existence and singularity structures of low regularity solutions to higher order degenerate hyperbolic equations, J. Differential Equations 256 (2014), 407-460.

[16] _ On the existence and cusp singularity of solutions to semilinear generalized Tricomi equations with discontinuous initial data, Commun. Contemp. Math. 17 (2015), 1450028 (49 pages).

[17] _ On the existence of low regularity solutions to semilinear generalized Tricomi equations in mixed type domains, J. Differential Equations 259 (2015), 7406-7462.

[18] H. Smith and D. Tataru, Sharp local well-posedness results for the nonlinear wave equation, Ann. of Math. (2) 162 (2005), 291-366.

[19] C. Sogge, Fourier integrals in classical analysis. Cambridge Tracts in Math., 105, Cambridge Univ. Press, Cambridge, 1993.

[20] E. Stein, Topics in harmonic analysis related to the Littlewood-Paley theory, Princeton Univ. Press, Princeton, 1970.

[21] M. Struwe, Semilinear wave equations, Bull. Amer. Math. Soc. 26 (1992), 53-85.

[22] K. Taniguchi and Y. Tozaki, A hyperbolic equation with double characteristics which has a solution with branching singularities, Math. Japon. 25 (1980), 279-300.

[23] F. Tricomi, Sulle equazioni lineari alle derivate parziali di secondo ordine di tipo misto, Memorie Accad. Lincei 5 (1923), 133-247.

[24] K. Yagdjian, A note on the fundamental solution for the Tricomi-type equation in the hyperbolic domain, J. Differential Equations 206 (2004), 227-252.

[25] _ Global existence for the n-dimensional semilinear Tricomi-type equations, Comm. Partial Differential Equations 31 (2006), 907-944.

[26] _ Integral transform approach to generalized Tricomi equations, J. Differential Equations 259 (2015), 59275981.

Department of Mathematics, NANJing University, NANJing 210093, China

E-mail address: zhuopingruan@nju.edu.cn

Mathematical Institute, University of Göttingen, Bunsenstr. 3-5, D-37073 Göttingen, Germany

E-mail address: iwitt@uni-math.gwdg.de

School of Mathematical Sciences, Nanjing Normal University, Nanjing 210023, China

E-mail address: huicheng@nju.edu.cn, $05407 @ n j n u . e d u . c n$ 